\title{
Decoherence and Thermalization of Quantum Spin Systems
}

\author{
Shengjun Yuan \\ Institute of Molecules and Materials, Radboud University of Nijmegen, 6525 ED Nijmegen, The Netherlands
}

\begin{abstract}
In this review, we discuss the decoherence and thermalization of a quantum spin system interacting with a spin bath environment, by numerically solving the time-dependent Schrödinger equation of the whole system. The effects of the topologic structure and the initial state of the environment on the decoherence of the two-spin and many-spin system are discussed. The role of different spinspin coupling is considered. We show under which conditions the environment drives the reduced density matrix of the system to a fully decoherent state, and how the diagonal elements of the reduced density matrix approach those expected for the system in the microcanonical or canonical ensemble, depending on the character of the additional integrals of motion. Our demonstration does not rely on time-averaging of observables nor does it assume that the coupling between system and bath is weak. Our findings show that the microcanonical distribution (in each eigenenergy subspace) and canonical ensemble are states that may result from pure quantum dynamics, suggesting that quantum mechanics may be regarded as the foundation of quantum statistical mechanics. Furthermore, our numerical results show that a fully decoherent quantum system prefers to stay in an equilibrium state with a maximum entropy, indicating the validity of the second law of thermodynamics in the decoherence process.
\end{abstract}

Keywords: Quantum Statistical Mechanics, Canonical Ensemble, Time-Dependent Schrödinger Equation, Decoherence, Second Law of Thermodynamics

\section{CONTENTS}

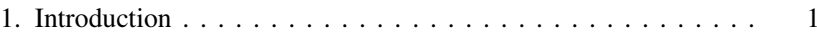

2. General Theory .................... 3

3. Quantum Spin System and Numerical Method . . . . . . . . . . 4

4. Decoherence of a Two-Spin System . . . . . . . . . . . . . . 5

4.1. A Two-Spin System . . . . . . . . . . . . . . . 5

4.2. Decoherence Without Energy Dissipation . . . . . . . . . . 7

4.3. Decoherence with Energy Dissipation . . . . . . . . . . 9

4.4. Summary . . . . . . . . . . . . . . . . . . . . . 10

5. Evolution to the Ground State of a Two-Spin System . . . . . 10

5.1. Symmetrical Coupling . . . . . . . . . . . . . . . . . 10

5.2. Antisymmetric Coupling . . . . . . . . . . . . . . . 12

5.3. Summary . . . . . . . . . . . . . . . . . . 14

6. Decoherence of a Many-Spin System . . . . . . . . . . . . . . . 15

6.1. Origin of the Microcanonical Distribution . . . . . . . . 15

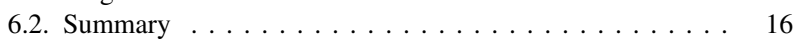

7. Thermalization of a Many-Spin System . . . . . . . . . . . . . 17

7.1. Origin of the Canonical Ensemble . . . . . . . . . . . 18

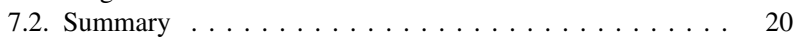

8. Conclusion and Discussion .............. 21

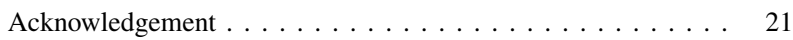

References ........................ 22

\section{INTRODUCTION}

The manner in which a quantum system becomes effectively classical is of great importance for the foundations of quantum physics. It has become increasingly clear that the symptoms of classicality of quantum systems can be induced by their environments. ${ }^{1}$

Intuitively, we expect that by turning on the interaction between the quantum system and the environment, the fluctuations in the environment will lead to a reduction of the coherence in the quantum system. This process is called decoherence..$^{2-11}$ The existence of decoherence in the quantum system represents a challenge for the realization of quantum computation and quantum information processing, ${ }^{12-15}$ which are expected to rely heavily on quantum coherence. In general, there are two different mechanisms that contribute to decoherence. If the environment is dissipative (or coupled to a dissipative system), the total energy is not conserved and the whole system relaxes to a stationary equilibrium state, for instance the thermal equilibrium state. In this review, we exclude this class of dissipative processes and restrict ourselves to closed systems in which a small quantum system is brought in contact with a much larger environment. Then, decoherence is solely due to the fact that the initial product state (wave function of the quantum system times wave function of the environment) evolves into an entangled state of the whole system. The interaction with the environment causes the 
initial pure state of the quantum system to evolve into a mixed state, described by a reduced density matrix, ${ }^{16}$ obtained by tracing out all the degrees of freedom of the environment. ${ }^{2-11,17,18}$

The decoherence programme is supposed to explain the macroscopic quantum superposition ("Schrödinger cat") paradox, that is, the inapplicability of the superposition principle to the macroworld. The states that are "robust" with respect to the interaction with the environment are called pointer states. ${ }^{9}$ If the Hamiltonian of the quantum system $H_{\mathrm{S}}$ is a perturbation, relative to the interaction Hamiltonian $H_{\mathrm{int}}$, the pointer states are eigenstates of $H_{\text {int }}{ }^{9-11}$ In this case, the pointer states are essentially "classical states," such as states with definite particle positions or with definite spin directions of individual particles for magnetic systems. In general, these classical "Schrödinger cat states," being a product state of individual particles forming the system, are not entangled.

On the contrary, if the interaction Hamiltonian $H_{\text {int }}$ is a perturbation, relative to the Hamiltonian of the quantum system $H_{\mathrm{S}}$, the pointer states are eigenstates of $H_{\mathrm{S}}{ }^{9,}{ }^{11} \mathrm{In}$ this case, the pointer states are not necessary classicaltype states, they may be "quantum" states such as standing waves, stationary electron states in atoms, tunneling-split states for a particle distributed between several potential wells, singlet or triplet states for magnetic systems, etc. ${ }^{11}$ This may explain, for example, that one can observe linear atomic spectra-the initial states of an atom under the equilibrium conditions are eigenstates of its Hamiltonian and not arbitrary superposition thereof. Some less trivial pointer states have been found in computer simulations of quantum spin systems for some range of the model parameters. ${ }^{20,21}$ In fact, the evolution to equilibrium of quantum spin systems is still an open issue. Recently, the effect of an environment of $N \gg 1$ spins on the entanglement of quantum spin systems has attracted much attention. ${ }^{20-42}$

Furthermore, recent simulation results of quantum spin systems show that different statistical ensembles such as the microcanonical (per eigenenergy subspace) and the canonical ensemble, could arise from the distribution of these pointer states (eigenstates) in the mixed state. ${ }^{26}$ This direct connection of quantum dynamics and statistical mechanics gives an explanation of a basic postulate in statistical mechanics: a generic "system" that interacts with a generic environment evolves into a state described by the canonical ensemble. Experience shows that this is true but a detailed understanding of this process, which is crucial for a rigorous justification of statistical physics and thermodynamics, is still lacking. ${ }^{43-64}$ In particular, in this context the meaning of "generic" is not clear. The key question is to what extent the evolution to the equilibrium state depends on the details of the dynamics of the whole system.

Earlier demonstrations of the fact that the system can be in the canonical ensemble state are based on the Ergodic averages, that the expectation values of the dynamical variables of the system approach their values for the subsystem that is in the thermal equilibrium state, ${ }^{43-46}$ or do not consider the dynamics of the system but assume that the state of the whole system has a special property called "canonical typicality," ${ }^{47-53}$ There are two basic assumptions in the derivation of the canonical typicality, one is that the whole system is in the microcanonical ensemble, another is that the interaction between the system and the environment is so small that it can be neglected. The theory of the canonical typicality is kinetic rather than dynamic, and it is yet unclear under which conditions the whole system will evolve into the region in Hilbert space where its subsystems are in the thermal equilibrium state. ${ }^{47}$ A very different setting to study nonequilibrium quantum dynamics is to start from an eigenstate of some initial Hamiltonian and push the system out of this state by a sudden change of the model parameters. ${ }^{54-60}$ To the best of our knowledge, it has not yet been shown that this approach leads to the establishment of the canonical equilibrium distribution. We also want to draw attention to the fact that a demonstration of relaxation to the canonical distribution requires a system with at least three different eigenenergies because a diagonal density matrix of a two-level system can always be represented as a canonical distribution..$^{61,62}$

In this review, we focus on recent results concerning the decoherence and thermalization in quantum spin systems. ${ }^{20,21,23-26}$ In Section 2, we give a general theory about decoherence and thermalization, and introduce several quantities that measure the effect of decoherence and

\section{Shengjun Yuan}


thermalization. In Section 3, we introduce the quantum spin model and the methods used in the numerical simulation. In Section 4, we focus on the decoherence of a two-spin quantum system, and consider the effect of different topological structures and different types of spinspin coupling. In Section 5, we discuss how a Heisenberg two-spin system evolves to the ground state. In Section 6, we consider the decoherence of many-spin systems. We show under which conditions the environment drives the reduced density matrix of the system to a fully decoherent state, which is described by the microcanonical distribution per eigenenergy subspace. In Section 7, by introducing the energy dissipation of the many-spin system, we show that the diagonal elements of the reduced density matrix approach those expected for the system in the canonical ensemble. Section 8 contains the conclusion and a brief discussion about the second law of thermodynamics in quantum systems.

\section{GENERAL THEORY}

In general, the state of a closed quantum system is described by a density matrix ${ }^{65,66} \rho$. The state of a quantum system interacting with an environment is represented by the reduced density matrix $\rho(t)$, obtained by tracing out all the degrees of freedom of the environment. Decoherence of the quantum system means that the amplitude of the off-diagonal terms in the reduced density matrix become smaller, and full decoherence corresponds to all off-diagonal terms being zero. Here we consider the case that the interaction Hamiltonian $H_{\text {int }}$ is a perturbation, but not necessary very small, relative to the Hamiltonian of the quantum system $H_{\mathrm{S}}$, and the reduced density matrix of the quantum system is represented in its energy eigenstates. The decoherence of the quantum system can also be monitored by the time dependence of the quadratic entropy $S_{\mathrm{S}}(t)=1-\operatorname{Tr} \rho^{2}(t)$ and the Loschmidt echo (reduced fidelity $)^{67-72} L(t)=\operatorname{Tr}\left(\rho(t) \rho_{0}(t)\right)$, where $\rho_{0}(t)$ is the density matrix for $H_{\text {int }}=0$.

The microcanonical ensemble is a mixed state where all accessible eigenstates have equal weight. The microcanonical distribution per eigenenergy subspace is characterized by a density matrix that is diagonal with respect to the eigenstates of the system Hamiltonian, and the diagonal elements which belong to the degenerate energy eigenstates are equal. A state with microcanonical distribution per eigenenergy subspace is a microcanonical ensemble if it has only one accessible eigenenergy.

The canonical ensemble is a mixed state where the diagonal elements take the form $\exp \left(-\beta E_{i}\right), \beta=1 / k_{B} T$ is proportional to the inverse of the temperature $T$ ( $k_{B}$ is Boltzmann's constant) and the $E_{i}$ 's denote the eigenenergies.

The distribution of the state of a quantum system is the microcanonical or canonical ensemble only if it is in a fully decoherent state.
The time evolution of a closed quantum system is governed by the time-dependent Schrödinger equation (TDSE). ${ }^{65,66}$ If the initial density matrix of an isolated quantum system is non-diagonal, then, according to the TDSE, its density matrix remains nondiagonal and never approaches the thermal equilibrium state with the canonical or microcanonical distribution. Therefore, in order to thermalize the system $S$, it is necessary to have the system $S$ interact with an environment $(E)$, also called the heat bath. Thus, the Hamiltonian of the whole system $(S+E)$ takes the form $H=H_{\mathrm{S}}+H_{\mathrm{E}}+H_{\mathrm{SE}}$, where $H_{\mathrm{S}}$ and $H_{\mathrm{E}}$ are the system and environment Hamiltonian, respectively, and $H_{\mathrm{SE}}$ describes the interaction between the system and the environment.

The state of the system $S$ is described by the reduced density matrix

$$
\rho(t) \equiv \operatorname{Tr}_{\mathrm{E}} \rho_{\mathrm{S}+\mathrm{E}}(t)
$$

where $\rho_{\mathrm{S}+\mathrm{E}}(t)$ is the density matrix of the whole system at time $t$ and $\mathbf{T r}_{\mathrm{E}}$ denotes the trace over the degrees of freedom of the environment.

The coherence of the system is conveniently characterized by $\sigma(t)$, which is a global measure for the size of the off-diagonal terms of the reduced density matrix, defined by

$$
\sigma(t)=\sqrt{\sum_{i=1}^{n-1} \sum_{j=i+1}^{n}\left|\rho_{i j}(t)\right|^{2}}
$$

Here $n$ denotes the dimension of the Hilbert space of system $S$ and $\rho_{i j}(t)$ is the matrix element $(i, j)$ of the reduced density matrix $\rho$ in the representation that diagonalizes $H_{\mathrm{S}}$. If $\sigma(t)=0$ the system is in a state of full decoherence.

The difference between the state $\rho(t)$ and a microcanonical distribution per eigenenergy subspace can be characterized by $\sigma(t)$ (measure of decoherence) and $\gamma(t)$ (measure of the difference between the diagonal terms corresponding to the degenerate eigenstates), which is defined by

$$
\gamma(t)=\sqrt{\sum_{i=1}^{n-1} \sum_{j=i+1}^{n}\left|\rho_{i i}(t)-\rho_{j j}(t)\right|^{2} \delta\left(E_{i}-E_{j}\right)}
$$

The system is in the microcanonical distribution per energy subspace if and only if $\sigma(t)=0$ and $\gamma(t)=0$.

The system $S$ is in its thermal equilibrium state only if the reduced density matrix takes the form

$$
\widehat{\rho} \equiv e^{-\beta H_{\mathrm{S}}} / \mathbf{T r}_{\mathrm{S}} e^{-\beta H_{\mathrm{S}}}
$$

where $\mathbf{T r}_{\mathrm{S}}$ denotes the trace over the degrees of freedom of the system $S$. The difference between the state $\rho(t)$ and the canonical distribution $\widehat{\rho}$ is represented by $\sigma(t)$ and $\delta(t)$, defined by

$$
\delta(t)=\sqrt{\sum_{i=1}^{n}\left(\rho_{i i}(t)-e^{-b(t) E_{i}} / \sum_{i=1}^{n} e^{-b(t) E_{i}}\right)^{2}}
$$


with

$$
b(t)=\frac{\sum_{i<j, E_{i} \neq E_{j}}\left[\ln \rho_{j j}(t)-\ln \rho_{i i}(t)\right] /\left(E_{j}-E_{i}\right)}{\sum_{i<j, E_{i} \neq E_{j}} 1}
$$

As the system relaxes to its canonical distribution both $\sigma(t)$ and $\delta(t)$ vanish, $b(t)$ converging to $\beta$.

\section{QUANTUM SPIN SYSTEM AND NUMERICAL METHOD}

Most theoretical investigations of decoherence have been carried out for oscillator models of the environment for which powerful path-integral techniques can be used to treat the environment analytically. ${ }^{17,18}$ On the other hand, it has been pointed out that a magnetic environment, described by quantum spins, is essentially different from the oscillator model in many aspects. ${ }^{19}$ For the simplest model of a single spin in an external magnetic field, some analytical results are known. ${ }^{19}$ For the generic case of two and more spins, numerical simulation ${ }^{20-26}$ is the main source of theoretical information.

A generic quantum spin model can be described by the Hamiltonian $H=H_{\mathrm{S}}+H_{\mathrm{E}}+H_{\mathrm{SE}}$ where

$$
\begin{gathered}
H_{\mathrm{S}}=-\sum_{i=1}^{N_{\mathrm{S}}-1} \sum_{j=i+1}^{N_{\mathrm{S}}} \sum_{\alpha=x, y, z} J_{i, j}^{\alpha} S_{i}^{\alpha} S_{j}^{\alpha} \\
H_{\mathrm{E}}=-\sum_{i=1}^{N-1} \sum_{j=i+1}^{N} \sum_{\alpha=x, y, z} \Omega_{i, j}^{\alpha} I_{i}^{\alpha} I_{j}^{\alpha} \\
H_{\mathrm{SE}}=-\sum_{i=1}^{N_{\mathrm{S}}} \sum_{j=1}^{N} \sum_{\alpha=x, y, z} \Delta_{i, j}^{\alpha} S_{i}^{\alpha} I_{j}^{\alpha}
\end{gathered}
$$

Here the $S^{\alpha}$ 's and $I^{\alpha}$ 's denote the spin-1/2 operators of the system and environment respectively (we use units such that $\hbar$ and $k_{\mathrm{B}}$ are one). An analytic solution of the TDSE can only be obtained for very special choices of the exchange integrals $J_{i, j}^{\alpha}, \Omega_{i, j}^{\alpha}$ and $\Delta_{i, j}^{\alpha}$ but it is straightforward to solve the TDSE numerically for any choice of the model parameters.

The state, that is the density matrix $\rho(t)$ of the whole system at time $t$, is completely determined by the choice of the initial state of the whole system and the numerical solution of the TDSE. In this review, the initial state of the whole system $(S+E)$ is a pure state. This state evolves in time according to

$$
|\Psi(t)\rangle=e^{-\mathrm{iH \textrm {t }}}|\Psi(0)\rangle=\sum_{i=1}^{n_{\mathrm{S}}} \sum_{p=1}^{n} c(i, p, t)|i, p\rangle
$$

where the states $\{|i, p\rangle\}$ denote a complete set of orthonormal states. In terms of the expansion coefficients $c(i, p, t)$, the reduced density matrix reads

$$
\begin{aligned}
\rho(t)_{i, j} & =\operatorname{Tr}_{\mathrm{E}} \sum_{p=1}^{n} \sum_{q=1}^{n} c^{*}(i, q, t) c(j, p, t)|j, p\rangle\langle i, q| \\
& =\sum_{p=1}^{n} c^{*}(i, p, t) c(j, p, t)
\end{aligned}
$$

which is easy to compute from the solution of the TDSE. We monitor the effects of decoherence by computing the matrix elements of the reduced density matrix $\rho(t)$ of the quantum system. As explained earlier, in the regime of interest $|\Delta| \ll|J|$, the pointer states are expected to be the eigenstates of the quantum systems. Hence, we compute the matrix elements of the density matrix in the basis of eigenvectors of the quantum system. We also compute the time dependence of the quadratic entropy $S_{\mathrm{S}}(t)=$ $1-\operatorname{Tr}^{2}(t)$ and the Loschmidt echo (reduced fidelity) ${ }^{67-72}$ $L(t)=\operatorname{Tr}\left(\rho_{0}(t) \rho(t)\right)$, where $\rho_{0}(t)$ is the density matrix for $H_{\mathrm{SE}}=0$. Another quantity of interest that can be extracted from the solution of the TDSE is the local density of states (LDOS)

$$
\begin{aligned}
\operatorname{LDOS}(E) & \equiv \frac{1}{2 \pi} \int_{-\infty}^{+\infty} d t e^{-i E t}\left\langle\Psi(0)\left|e^{-i H t}\right| \Psi(0)\right\rangle \\
& =\sum_{k=1}^{D}\left|\left\langle\Psi(0) \mid \varphi_{k}\right\rangle\right|^{2} \delta\left(E-E_{k}\right)
\end{aligned}
$$

where $D=n+n_{\mathrm{S}},\left\{\left|\varphi_{k}\right\rangle\right\}$, and $\left\{E_{k}\right\}$ denote the dimension of the Hilbert space, the eigenstates and eigenvalues of the whole system, respectively. The LDOS is "local" with respect to the initial state: It provides information about the overlap of the initial state and the eigenstates of $H$.

The notation to specify the initial state is as follows: (1) $|G R O U N D\rangle_{\mathrm{S}}$ is the ground state or a random superposition of all degenerated ground states of the system;

(2) $|G R \widetilde{O U N D}\rangle_{\mathrm{S}}$ is the state which has the energy close but not equal to that of the ground state;

(3) $|U U\rangle_{\mathrm{S}}$ is a state in which all spins of the system are up, meaning that in this state, the expectation value of each spin is one;

(4) $|U D\rangle_{S}$ is a state in which two nearest-neighbor spins of the system are antiparallel implying that in this state, the correlation of their $z$-components is minus one;

(5) $|\widetilde{U D}\rangle_{\mathrm{S}}$ is a state close to $|U D\rangle_{\mathrm{S}}$, but the correlation of their $z$-components is larger than minus one;

(6) $|R R\rangle_{\mathrm{S}}$ denotes the product state of random superpositions of the states of the individual spins of the system;

(7) $|R A N D O M\rangle_{\mathrm{S}}$ denotes a random superposition of all possible basis states.

The same notation is used for the spins in the environment, the subscript $S$ being replaced by $E$.

As we report results for many different types of spin systems it is useful to introduce a simple terminology to classify them according to symmetry and connectivity. The terms "XY," "Heisenberg," "Heisenberg-type," "Ising," "Ising-type" and Ising \pm system refer to the following cases:

(1) XY: $J_{i, j}^{x}=J_{i, j}^{y}=J$ and $J_{i, j}^{z}=0$;

(2) Heisenberg: $J_{i, j}^{x}=J_{i, j}^{y}=J_{i, j}^{z}=J$;

(3) Heisenberg-type: $J_{i, j}$ are uniform random in the range $[-|J|,|J|]$;

(4) Ising: $J_{i, j}^{x}=J_{i, j}^{y}=0$ and $J_{i, j}^{z}=J$; 
(5) Ising-type: $J_{i, j}^{x}=J_{i, j}^{y}=0$ and $J_{i, j}^{z}$ are uniform random in the range $[-|J|,|J|]$;

(6) Ising $\pm: J_{i, j}^{x}=J_{i, j}^{y}=0$ and $J_{i, j}^{z}$ are random $-|J|$ or $|J|$.

The same terminology is used for the Hamiltonian $H_{\mathrm{E}}$ of the environment and for the interaction Hamiltonian $H_{\mathrm{SE}}$. In our model, all the spins of the system interact with each spin of the environment. To characterize the connectivity $K$ of the spins within the system (environment), we use the term "ring" $(K=2)$ for spins forming a onedimensional chain with nearest-neighbor interactions and periodic boundary conditions, "square-lattice" $(K=4)$ or "triangular-lattice" $(K=6)$ if the spins are located on a two-dimensional square or triangular lattice with nearestneighbor interactions, and "spin glass" $(K=N-1)$ when all the spins within the system (environment) interact with each other with a Heisenberg-type interaction. ${ }^{73,74}$

The time evolution of the whole system is obtained by solving the TDSE for the many-body wave function $|\Psi(t)\rangle$, describing the system plus the environment. ${ }^{78-90}$ The numerical method that we use is described in Ref. [78]. It is based on the numerically exact Chebyshev polynomial decomposition of the operator $U(t)=e^{-\mathrm{itH}}$. It is very efficient and conserves the energy of the whole system to machine precision. It is widely used in the simulation of quantum spin systems, such as the study of decoherence, ${ }^{20,21,23-26}$ the modeling of a quantum computer, ${ }^{89}$ the propagation of the quantum spin wave, ${ }^{91}$ and the study of stability of the quantum domain wall, ${ }^{92}$ etc.

The simulation of the wave function is performed in the spin-up and spin-down basis, and it is convenient to use this basis to apply the Hamiltonian operator on the wave vector, which is a basic operation in the Chebyshev polynomial algorithm. Based on the wave function of the whole system in the up-down basis, we first calculate the reduced density matrix of the quantum system by tracing out the degrees of freedom of the environment, then diagonalize the Hamiltonian $H_{\mathrm{S}}$ to get all the energy eigenvalues and the corresponding eigenstates of the quantum system, and finally transform the reduced density matrix from the updown basis to the energy eigenstate basis. With the reduced density matrix we can calculate all the physical quantities of the quantum system, such as the energy, the entropy, the measure of decoherence $\sigma$, the measure of the distribution $\gamma, \delta$ and the effective inverse temperature $b$, etc.

\section{DECOHERENCE OF A TWO-SPIN SYSTEM}

\subsection{A Two-Spin System}

The quantum state of a two-spin system is completely determined by its reduced $4 \times 4$ density matrix. Although the reduced density matrix contains all the information about the quantum system, it is often convenient to characterize the state of the two-spin system by other quantities such as the correlation functions $\left\langle\mathbf{S}_{1} \cdot \mathbf{S}_{2}\right\rangle,\left\langle S_{1}^{z} S_{2}^{z}\right\rangle$, and $\left\langle S_{1}^{x} S_{2}^{x}\right\rangle$, the single-spin magnetizations $\left\langle S_{1}^{x}\right\rangle,\left\langle S_{2}^{x}\right\rangle$, and $M \equiv\left\langle S_{1}^{z}+S_{2}^{z}\right\rangle$, and the concurrence $C$. ${ }^{94,95}$ In Table I, we show the values of these quantities for different states of the two-spin system.

The concurrence, which is a convenient measure for the entanglement of the two spins is defined as ${ }^{94,95}$

$$
C(\rho)=\max \left(0, \lambda_{1}-\lambda_{2}-\lambda_{3}-\lambda_{4}\right)
$$

where the $\lambda_{i}$ are the eigenvalues, in decreasing order, of the Hermitian matrix

$$
R \equiv \sqrt{\sqrt{\rho} \widetilde{\rho} \sqrt{\rho}}
$$

Here $\rho$ is the reduced density matrix of central spin pairs based on the standard basis $|\uparrow\rangle|\uparrow\rangle,|\uparrow\rangle|\downarrow\rangle,|\downarrow\rangle|\uparrow\rangle$, $|\downarrow\rangle|\downarrow\rangle$, and

$$
\tilde{\rho}=\left(\sigma_{y} \otimes \sigma_{y}\right) \rho^{*}\left(\sigma_{y} \otimes \sigma_{y}\right)
$$

where

$$
\sigma_{y}=\left(\begin{array}{cc}
0 & -i \\
i & 0
\end{array}\right)
$$

and $\rho^{*}$ is the complex conjugate of $\rho$.

In fact, the concurrence $C$ is a measure between the state $|\psi\rangle$ and the state with the two spins flipped $|\widetilde{\psi}\rangle$ :

$$
C=|\langle\psi \mid \widetilde{\psi}\rangle|
$$

The singlet state, $|\psi\rangle=(|\uparrow\rangle|\downarrow\rangle-|\downarrow\rangle|\uparrow\rangle) / \sqrt{2}$ is unchanged under flipping two spins, therefore $C=1$. The triplet state $|\psi\rangle=(|\uparrow\rangle|\downarrow\rangle+|\downarrow\rangle|\uparrow\rangle) / \sqrt{2}$ is also unchanged under flipping two spins, so $C=1$. For $|\uparrow\rangle|\uparrow\rangle$, $|\uparrow\rangle|\downarrow\rangle,|\downarrow\rangle|\uparrow\rangle$, and $|\downarrow\rangle|\downarrow\rangle$, the state is totally different if the two spins flip and then $C=0$.

In the case that the two spins are coupled by the isotropic Heisenberg interaction, the Hamiltonian of the system simplifies

$$
H_{\mathrm{S}}=-J \mathbf{S}_{1} \mathbf{S}_{2}
$$

Table I. The values of the correlation functions $\left\langle\mathbf{S}_{1} \cdot \mathbf{S}_{2}\right\rangle,\left\langle S_{1}^{z} S_{2}^{z}\right\rangle$, $\left\langle S_{1}^{x} S_{2}^{x}\right\rangle$, the total magnetization $M$, the concurrence $C$ and the magnetization $\left\langle S_{1}^{z}\right\rangle$ for different states of the two-spin quantum system.

\begin{tabular}{lcccccc}
\hline$|\varphi\rangle$ & $\left\langle\mathbf{S}_{1} \cdot \mathbf{S}_{2}\right\rangle$ & $\left\langle S_{1}^{z} S_{2}^{z}\right\rangle$ & $\left\langle S_{1}^{x} S_{2}^{x}\right\rangle$ & $M$ & $C$ & $\left\langle S_{1}^{x}\right\rangle$ \\
\hline$(|\uparrow \downarrow\rangle-|\downarrow \uparrow\rangle) / \sqrt{2}$ & $-3 / 4$ & $-1 / 4$ & $-1 / 4$ & 0 & 1 & 0 \\
$(|\uparrow \downarrow\rangle+|\downarrow \uparrow\rangle) / \sqrt{2}$ & $1 / 4$ & $-1 / 4$ & $1 / 4$ & 0 & 1 & 0 \\
$(|\uparrow \uparrow\rangle-|\downarrow \downarrow\rangle) / \sqrt{2}$ & $1 / 4$ & $1 / 4$ & $-1 / 4$ & 0 & 1 & 0 \\
$(|\uparrow \uparrow\rangle+|\downarrow \downarrow\rangle) / \sqrt{2}$ & $1 / 4$ & $1 / 4$ & $1 / 4$ & 0 & 1 & 0 \\
$|\uparrow \downarrow\rangle$ & $-1 / 4$ & $-1 / 4$ & 0 & 0 & 0 & $1 / 2$ \\
$|\downarrow \uparrow\rangle$ & $-1 / 4$ & $-1 / 4$ & 0 & 0 & 0 & $-1 / 2$ \\
$|\uparrow \uparrow\rangle$ & $1 / 4$ & $1 / 4$ & 0 & 1 & 0 & $1 / 2$ \\
$|\downarrow \downarrow\rangle$ & $1 / 4$ & $1 / 4$ & 0 & -1 & 0 & $-1 / 2$ \\
\hline & & & & & &
\end{tabular}


and the four eigenstates of $H_{\mathrm{S}}$ are given by

$$
\begin{gathered}
\left|T_{1}\right\rangle=|\uparrow \uparrow\rangle=|1\rangle \\
|S\rangle=\frac{|\uparrow \downarrow\rangle-|\downarrow \uparrow\rangle}{\sqrt{2}}=|2\rangle \\
\left|T_{0}\right\rangle=\frac{|\uparrow \downarrow\rangle+|\downarrow \uparrow\rangle}{\sqrt{2}}=|3\rangle \\
\left|T_{-1}\right\rangle=|\downarrow \downarrow\rangle=|4\rangle
\end{gathered}
$$

satisfying

$$
H_{\mathrm{S}}|S\rangle=E_{\mathrm{S}}|S\rangle, H_{\mathrm{S}}\left|T_{1,0,-1}\right\rangle=E_{\mathrm{T}}\left|T_{1,0,-1}\right\rangle
$$

where $E_{\mathrm{S}}=3 J / 4$ and $E_{\mathrm{T}}=-J / 4$.

From Table I, it is clear that the singlet state $|S\rangle$ is the most easily distinguished state as the two-spin system is in the singlet state if and only if $\left\langle\mathbf{S}_{1} \cdot \mathbf{S}_{2}\right\rangle=-3 / 4$. To identify the other states, we usually need to know at least two of the quantities listed in Table I. For example, to make sure that the system is in the triplet state $\left|T_{0}\right\rangle$, the values of $\left\langle\mathbf{S}_{1} \cdot \mathbf{S}_{2}\right\rangle$ and $\left\langle S_{1}^{z} S_{2}^{z}\right\rangle$ should match with the corresponding entries of Table I. Likewise, the two-spin system will be in the state $|\uparrow \uparrow\rangle$ if $\left\langle\mathbf{S}_{1} \cdot \mathbf{S}_{2}\right\rangle=1 / 4$ and $M=1$.

If the interaction between the Heisenberg quantum system and the environment is isotropic, that is, if $\Delta_{i, j}^{(x)}=$ $\Delta_{i, j}^{(y)}=\Delta_{i, j}^{(z)} \equiv \Delta$ for all $i, j$, then the Hamiltonian $H_{\mathrm{SE}}$ is simplified as

$$
H_{\mathrm{SE}}=-\Delta\left(\mathbf{S}_{1}+\mathbf{S}_{2}\right) \cdot \sum_{j=1}^{N} \mathbf{I}_{j}
$$

which leads to $\left[H_{\mathrm{S}}, H_{\mathrm{SE}}\right]=0$. As shown in Ref. [24], if the energy of the quantum system is conserved, then the decoherence process is determined by $H_{\mathrm{SE}}, H_{\mathrm{E}}$, the initial state of whole system $\left|\Psi\left(t_{0}\right)\right\rangle$, and the eigenstates of the quantum system. In other words, in this case, $L(t)$ and $|\rho(t)|$ do not depend on $J$, which means that the relative value of $\Delta / J$ has no effect on the decoherence process. Furthermore, if we take the interactions between the environment spins to be isotropic, that is, $\Omega_{i, j}^{(x)}=\Omega_{i, j}^{(y)}=\Omega_{i, j}^{(z)} \equiv \Omega_{i, j}$ for all $i, j$, then the Hamiltonian

$$
H_{\mathrm{E}}=-\sum_{i=1}^{N-1} \sum_{j=i+1}^{N} \Omega_{i, j} \mathbf{I}_{i} \cdot \mathbf{I}_{j}
$$

commutes with $H_{\mathrm{SE}}$, and therefore $H_{\mathrm{E}}$ has also no effect on the decoherence process.

In fact, since $\left[H_{\mathrm{S}}, H_{\mathrm{SE}}\right]=0$, the time evolution operator of the whole system $e^{-\mathrm{iHt}}$ can be represented as $e^{-\mathrm{iH}_{\mathrm{S}} \mathrm{t}} e^{-i\left(H_{\mathrm{SE}}+H_{\mathrm{E}}\right) t}$. The initial state of the quantum system can be represented as $\left|\varphi\left(t_{0}\right)\right\rangle=\sum_{\mathrm{k}} a_{\mathrm{k}}|k\rangle$, where $\{|k\rangle\}$ and $\left\{E_{\mathrm{k}}\right\}$ are the eigenstates and corresponding eigenvalues of the quantum system, that is, $H_{\mathrm{S}}|k\rangle=E_{\mathrm{k}}|k\rangle$.

For an isolated quantum system $\left(H_{\mathrm{SE}}=0\right)$, the time evolution of the density matrix of the quantum system is given by

$$
\rho_{0}(t)=\sum_{\mathrm{k}, 1} e^{-i\left(E_{\mathrm{k}}-E_{1}\right) t} a_{\mathrm{k}} a_{l}^{*}|k\rangle\langle l|
$$

If the quantum system is coupled to a bath with initial state $\phi\left(t_{0}\right)$, the state of the whole system at time $t$ is given by

$$
\begin{aligned}
|\Psi(t)\rangle & =e^{-i H t}\left|\Psi\left(t_{0}\right)\right\rangle \\
& =\sum_{k} e^{-i E_{\mathrm{k}} t} a_{\mathrm{k}} e^{-i\left(H_{\mathrm{SE}}+H_{\mathrm{E}}\right) t}|k\rangle\left|\phi\left(t_{0}\right)\right\rangle
\end{aligned}
$$

As $\left[H_{\mathrm{S}}, H_{\mathrm{SE}}\right]=0$, we have $H_{\mathrm{SE}}|k\rangle\left|\phi\left(t_{0}\right)\right\rangle=|k\rangle M_{\mathrm{k}}\left|\phi\left(t_{0}\right)\right\rangle$ and hence, the state at time $t$ becomes

$$
|\Psi(t)\rangle=\sum_{\mathrm{k}} a_{\mathrm{k}} e^{-i E_{\mathrm{k}} t}|k\rangle\left|\phi_{\mathrm{k}}(t)\right\rangle
$$

where

$$
\left|\phi_{k}(t)\right\rangle \equiv e^{-i\left(M_{\mathrm{k}}+H_{\mathrm{E}}\right) t}\left|\phi\left(t_{0}\right)\right\rangle
$$

The density matrix $\rho_{S+E}(t)$ of the whole system is

$$
\begin{aligned}
\rho_{\mathrm{S}+\mathrm{E}}(t) & =|\Psi(t)\rangle\langle\Psi(t)| \\
& =\sum_{\mathrm{k}, 1} e^{-i\left(E_{\mathrm{k}}-E_{1}\right) t} a_{\mathrm{k}} a_{1}^{*}|k\rangle\left|\phi_{\mathrm{k}}(t)\right\rangle\langle l|\left\langle\phi_{1}(t)\right|
\end{aligned}
$$

and the reduced density matrix $\rho(t)$ of the quantum system is

$$
\begin{aligned}
\rho(t) & =\operatorname{Tr}_{\mathrm{E}} \rho_{\mathrm{S}+\mathrm{E}}(t) \\
& =\sum_{\mathrm{k}, 1} e^{-i\left(E_{\mathrm{k}}-E_{1}\right) t} a_{\mathrm{k}} a_{1}^{*}\left\langle\phi_{1}(t) \mid \phi_{\mathrm{k}}(t)\right\rangle|k\rangle\langle l|
\end{aligned}
$$

The Loschmidt echo (reduced fidelity) $L(t)$ of the quantum system can be calculated as

$$
\begin{aligned}
L(t)= & \operatorname{Tr}\left(\rho(t) \rho_{0}(t)\right) \\
= & \operatorname{Tr}\left[\sum_{\mathrm{k}, 1} e^{-i\left(E_{\mathrm{k}}-E_{1}\right) t} a_{\mathrm{k}} a_{1}^{*}\left\langle\phi_{l}(t) \mid \phi_{\mathrm{k}}(t)\right\rangle|k\rangle\langle l|\right. \\
& \left.\quad \times \sum_{\mathrm{m}, \mathrm{n}} e^{-i\left(E_{\mathrm{m}}-E_{\mathrm{n}}\right) t} a_{\mathrm{m}} a_{\mathrm{n}}^{*}|m\rangle\langle n|\right] \\
= & \sum_{\mathrm{k}, 1}\left|a_{\mathrm{k}}\right|^{2}\left|a_{1}\right|^{2}\left\langle\phi_{1}(t) \mid \phi_{k}(t)\right\rangle
\end{aligned}
$$

It is clear that if $\left[H_{\mathrm{S}}, H_{\mathrm{SE}}\right]=0$, the decoherence process is determined by the initial state of the quantum system $\left\{a_{\mathrm{k}}\right\}$ and the time evolution of $\left\{\left|\phi_{\mathrm{k}}(t)\right\rangle\right\}$. As shown in Eq. (24), the $\left\{\left|\phi_{\mathrm{k}}(t)\right\rangle\right\}$ are determined by the initial state of the bath $\phi\left(t_{0}\right)$, the eigenstates $\{|k\rangle\}$ of the quantum system, and the Hamiltonians $H_{\mathrm{SE}}$ and $H_{\mathrm{E}}$. The eigenvalues $\left\{E_{\mathrm{k}}\right\}$ have no effect on the decoherence process. Thus, multiplying $H_{\mathrm{S}}$ by a constant does not change $L(t)$ and the diagonal elements of the reduced density matrix $\rho_{\mathrm{S}}(t)$. The time evolution of the absolute value of the off-diagonal elements

$$
\left|\rho_{\mathrm{S}}(t)_{\mathrm{kl}}\right|=\left|a_{\mathrm{k}} a_{1}^{*}\right|\left\langle\phi_{1}(t) \mid \phi_{\mathrm{k}}(t)\right\rangle
$$

is independent of $H_{\mathrm{S}}$. This means that the relevant values of the coupling between the system spins $(J)$ and between the system and the environment $(\Delta)$ have no effect on the decoherence process. 
If $\left[H_{\mathrm{S}}, H_{\mathrm{SE}}\right]=0$ and $\left[H_{\mathrm{SE}}, H_{\mathrm{E}}\right]=0$, then, Eq. (24) becomes

$$
\left|\phi_{k}(t)\right\rangle=e^{-i M_{k} t} e^{-i H_{\mathrm{E}} t}\left|\phi\left(t_{0}\right)\right\rangle
$$

and therefore we have

$$
\left\langle\phi_{l}(t) \mid \phi_{k}(t)\right\rangle=\left\langle\phi\left(t_{0}\right)\left|e^{-i\left(M_{\mathrm{k}}-M_{1}\right) t}\right| \phi\left(t_{0}\right)\right\rangle
$$

implying that $\left|\rho_{\mathrm{S}}(t)_{\mathrm{kl}}\right|$ and $L(t)$ do not depend on $H_{\mathrm{E}}$.

Our goal is to find under which conditions the quantum system can evolve into a classical mixed state, that is, the elements in the reduced density matrix satisfying

$$
\begin{aligned}
& \left|\rho_{\mathrm{S}}(t)_{k l}\right|=0 \text { if } k \neq l \\
& \left|\rho_{\mathrm{S}}(t)_{k l}\right| \neq 0 \text { if } k=l
\end{aligned}
$$

\subsection{Decoherence Without Energy Dissipation}

In previous work, ${ }^{23-26}$ it was shown that a frustrated environment, such as described by a Heisenberg-type $H_{\mathrm{E}}$, can enhance the decoherence of the quantum system. The typical results of the (full) decoherence in the Heisenberg twospin system without energy dissipation are the following.

In Figures 2 and 3, we show the time evolution of the elements of the reduced density matrix $\rho(t)$ for different connectivity $K$ with same $\Omega$, or different $\Omega$ with same $K$, for the case $\left[H_{\mathrm{S}}, H_{\mathrm{SE}}\right]=0$.

If $|\Delta| \gg \Omega \sqrt{K}$, in the absence of interactions between the environment spins $(\Omega \sqrt{K}=0)$ and after the initial decay, the quantum system exhibits long-time oscillations (see Fig. 2(a)(left)). As shown in Refs. [20, 22], in the limit of a large environment $(N \rightarrow \infty)$

$$
\operatorname{Re} \rho_{23}(t)=\left[\frac{1}{6}+\frac{1-b t^{2}}{3} e^{-c t^{2}}\right] \cos \omega t
$$

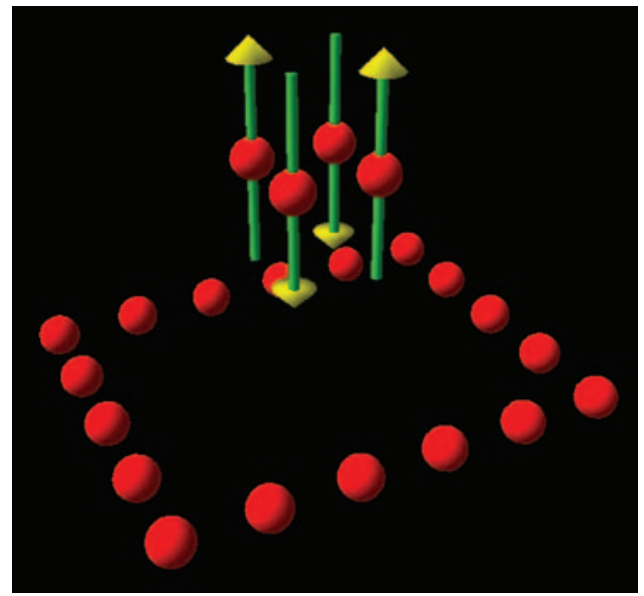

Fig. 1. A typical configuration of a quantum spin system surrounded by a quantum spin bath $\left(N_{\mathrm{S}}=4\right.$ and $\left.N=18\right)$. The quantum spin system consists of four spin $1 / 2$ particles, with orientation spin-up or spin-down. The bath spins are in a complicated random superposition state for which the expectation values $\left\langle S_{x}\right\rangle,\left\langle S_{y}\right\rangle$ and $\left\langle S_{z}\right\rangle$ of each spin are all zero. where $b=N \Delta^{2} / 4, c=b / 2$ and $\omega=J-\Delta$. Equation (32) clearly shows the two-step process, that is, after the initial Gaussian decay of the amplitude of the oscillations, the oscillations revive and their amplitude levels of by a factor of $1 / 3$ (see Ref. [22]). Due to conservation laws, this behavior does not change if we introduce an isotropic Heisenberg Hamiltonian in the environment $\left(\Omega_{i, j}^{(\alpha)} \equiv \Omega\right.$ for all $\alpha, i$ and $j$ ), independent of $K$. This is also confirmed by our numerical results (not shown).

If $|\Delta| \approx \Omega \sqrt{K}$, the initial Gaussian decay of the quantum system is not sensitive to the presence of a Heisenberg-type environment $H_{\mathrm{E}}$, but there is a decay of the amplitude of the long-living oscillations. The larger $K$ (see Figs. 2(b-e)(left)) or $\Omega$ (see Figs. 3(a, c)), the faster the decay is.

If $|\Delta| \ll \Omega \sqrt{K}$ and $\Omega$ is comparable with $J$, keeping $K$ fixed and increasing $\Omega$ smoothly changes the initial decay from Gaussian (fast) to exponential (slow). The long-living oscillations are completely suppressed (see Figs. 3(b, d)). For large $\Omega$, the simulation data fits very
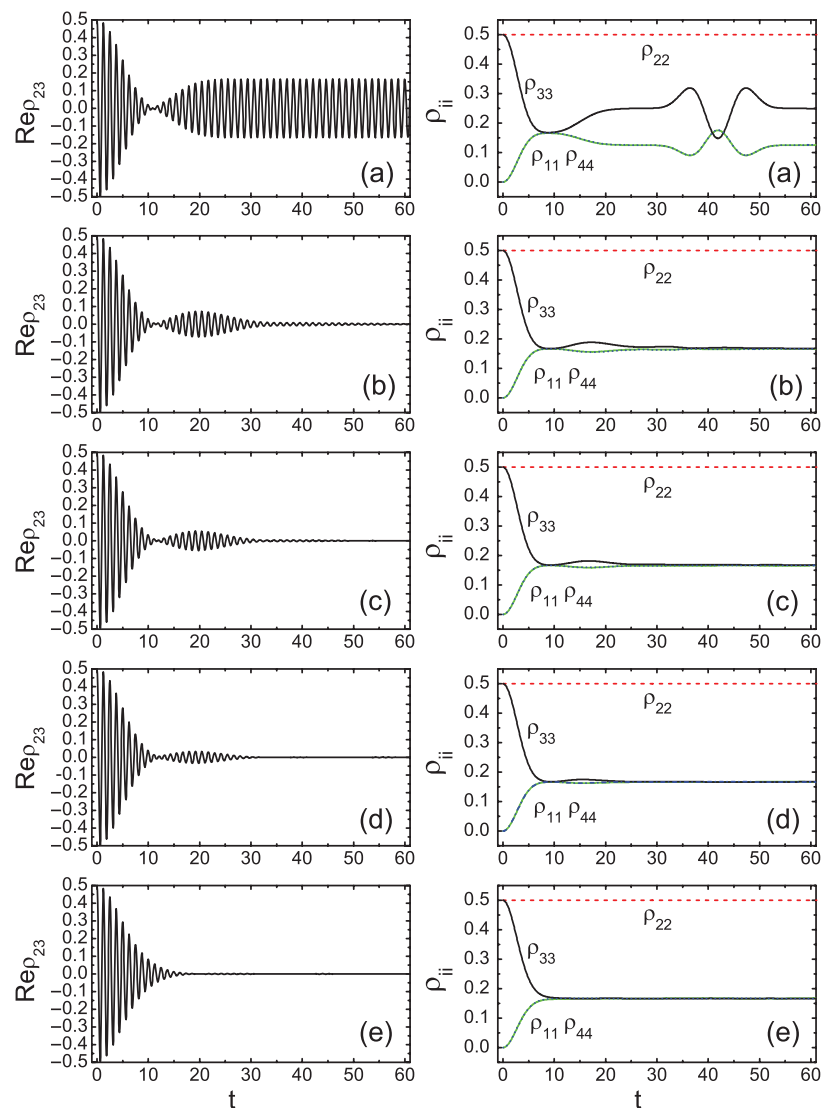

Fig. 2. Time evolution of the real part of the off-diagonal element $\rho_{23}$ (left panel) and the diagonal elements $\rho_{11}, \ldots, \rho_{44}$ (right panel) of the reduced density matrix of a Heisenberg two-spin system $(J=-5)$, coupled via an isotropic Heisenberg interaction $H_{\mathrm{SE}}(\Delta=-0.075)$ to a Heisenberg-type environment $H_{\mathrm{E}}(\Omega=0.1)$ with different connectivity: (a) $K=0$; (b) $K=2$; (c) $K=4$; (d) $K=6$; (e) $K=N-1$. Reprinted with permission from [25], S. Yuan et al., Phys. Rev. B 77, 184301 (2008). (c) 2008. 

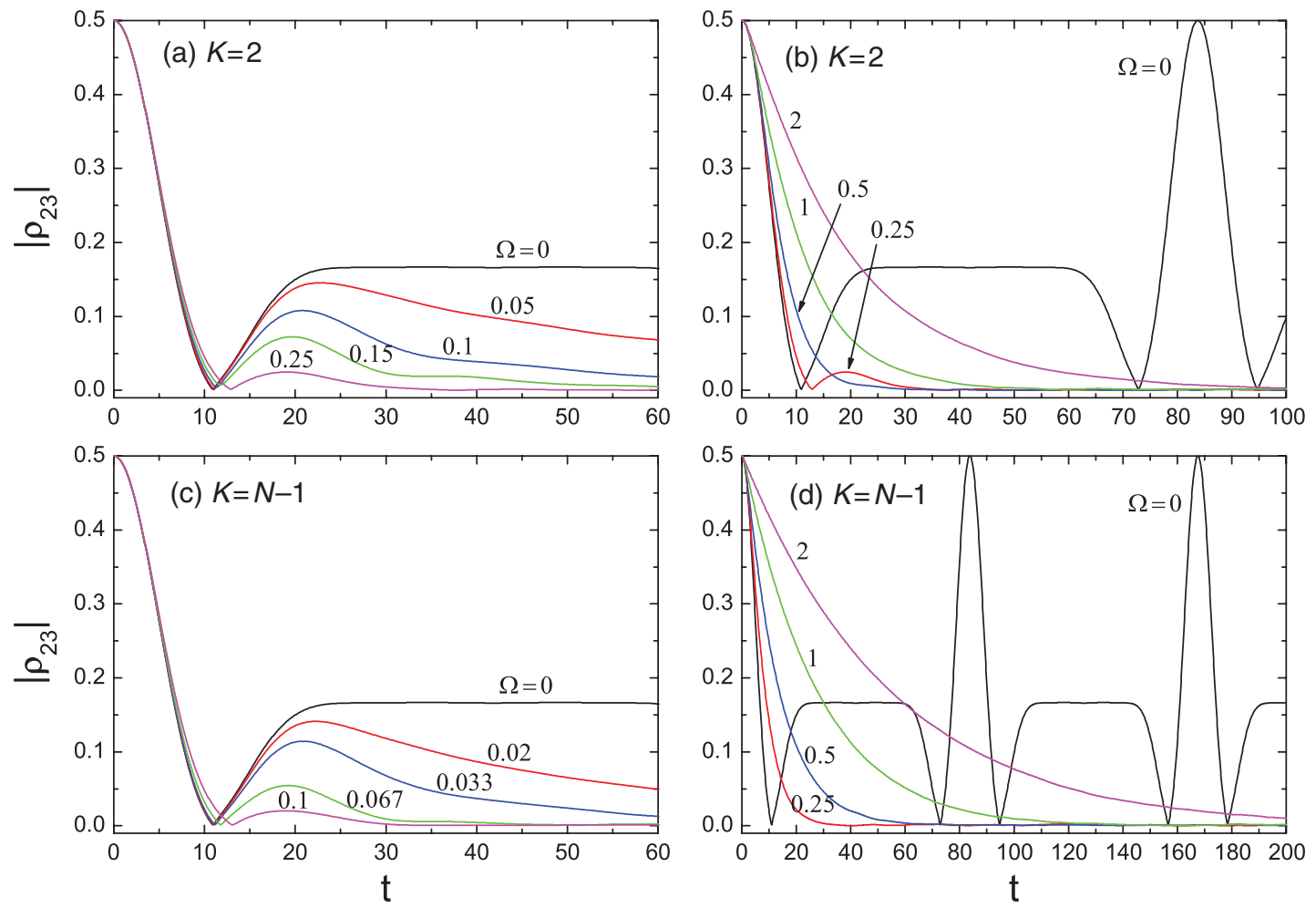

Fig. 3. Time evolution of the off-diagonal element $\rho_{23}$ of the reduced density matrix of a Heisenberg two-spin system $(J=-5)$, interacting with a Heisenberg-type environment $H_{\mathrm{E}}$ via an isotropic Heisenberg Hamiltonian $H_{\mathrm{SE}}(\Delta=-0.075)$ for the same geometric structures in the environment: (a, b) $K=2$ and (c, d) $K=N-1$. The number next to each curve is the corresponding value of $\Omega$. Reprinted with permission from [25], S. Yuan et al., Phys. Rev. B 77, 184301 (2008). (c) 2008.

well to

$$
\left|\rho_{23}(t)\right|=\frac{1}{2} e^{-A_{K}(\Omega) t}
$$

where $A_{\mathrm{K}}(\Omega)$ is approximately linearly dependent on $\Omega$ :

$$
A_{\mathrm{K}}(\Omega) \approx \Omega \widetilde{A}_{\mathrm{K}}
$$

and we find that $\widetilde{A}_{2}=9.13$ and $\widetilde{A}_{N-1}=26.73$.

Physically, the observed behavior can be understood as follows. $^{25}$ If $|\Delta| \approx \Omega \sqrt{K}$, a bath spin is roughly equally affected by the motion of the other bath spins and the system spins. Therefore, each bath spin follows the original dynamics, as if there was no coupling between bath spins. This explains why the initial Gaussian decay is insensitive to the values of $K$ or $\Omega$. After the initial decay, the whole system is expected to reach a stationary state, but because of the presence of Heisenberg-type interactions between the bath spins, it leads to a decrease of the coherence between the singlet and triplet states, and therefore a new stationary state of the bath is established, suppressing the long-living oscillations.

For larger $K$, the distance between two bath spins, defined as the minimum number of bonds connecting the two spins, becomes smaller. For instance, for $K=2$, this distance is $(N-2) / 2$, and for $K=N-1$, it is zero. For fixed $\Omega$ and larger $K$ the fluctuations in the spin bath propagate faster, and therefore the evolution to the stationary state is faster. Furthermore, since the environment in our model is a highly frustrated system, increasing the connectivity $K$ will increase the energy resolution of the eigenstates, which makes the dynamics of the environment more complicated. For fixed $K$, increasing the coupling strength between the bath spins will speed up the dynamics of the bath, that is, the larger $\Omega$ the faster will be the evolution to the stationary state. However the coupling strength within the environment should not be too large, because otherwise the energy resolution in the bath will be too small to lead the energy dissipation of the quantum system.

In the opposite case $|\Delta| \ll \Omega \sqrt{K}$ and $\Omega$ is comparable with $J, H_{\mathrm{SE}}$ is a small perturbation relative to $H_{\mathrm{E}}$ and the coupling between the bath spins is the dominant factor in determining the dynamics of the bath spins. Therefore, by increasing $K$ or $\Omega$, the bath spins will have less freedom to follow the dynamics induced by the coupling to the two system spins, the influence of the bath on the quantum system will decrease, and the (exponential) decay will become slower.

Here we have compared $\Omega \sqrt{K}$ to $|\Delta|$ to distinguish different regimes. As a matter of fact, $\Omega \sqrt{K}$ does not completely characterize the decoherence process, but it can be used to characterize its time scale. Indeed, as shown in Figure 4, for different $\sqrt{K}$ and $\Omega$ but the same value of $\Omega \sqrt{K}$, the time evolution of $L(t)$ is very similar. Note that 

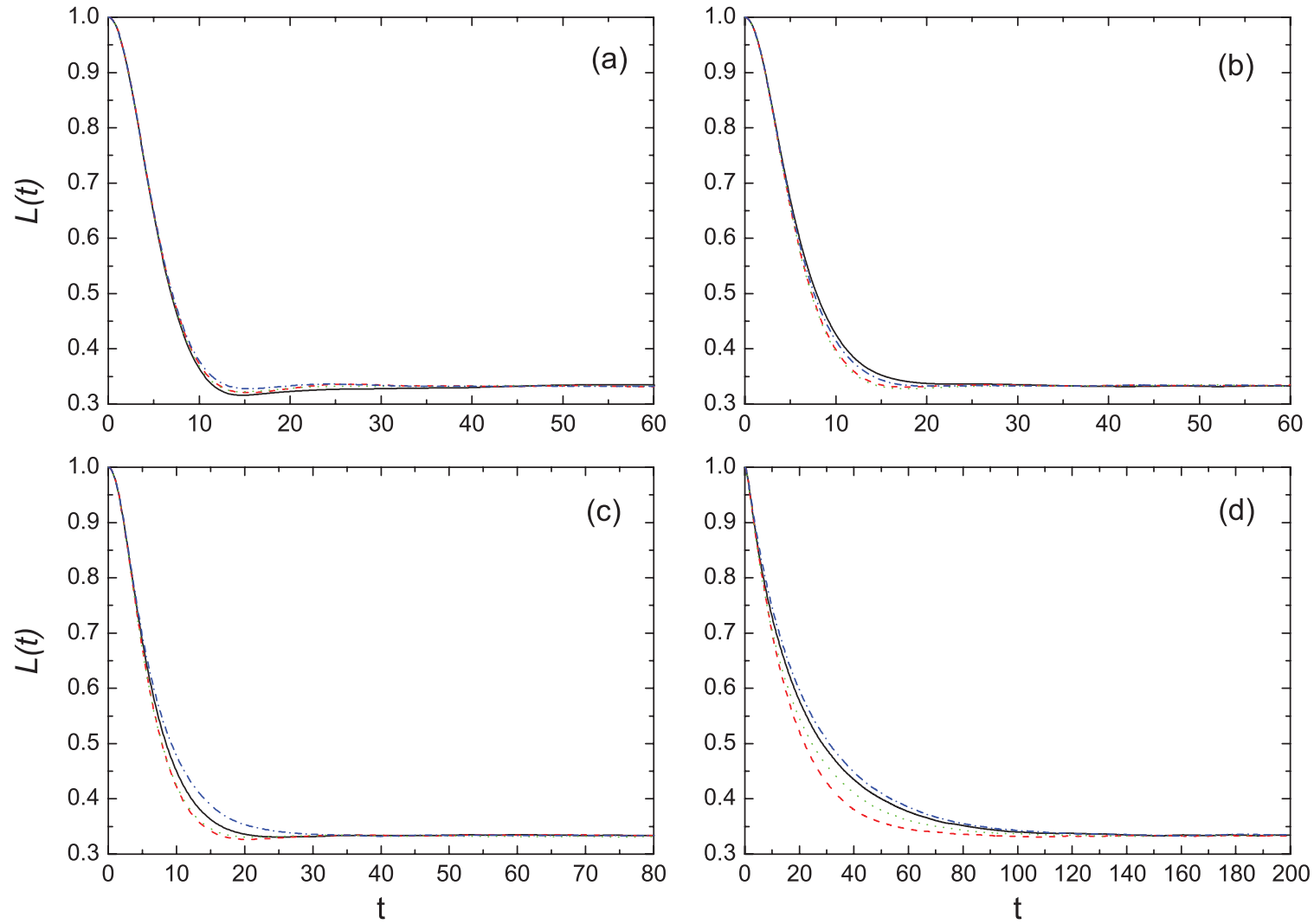

Fig. 4. Time evolution of the Loschmidt echo $L(t)$ of a Heisenberg two-spin system $(J=-5)$, interacting with a Heisenberg-type environment $H_{\mathrm{E}}$ via a Heisenberg $(\Delta=-0.075)$ Hamiltonian $H_{\mathrm{SE}}$. The values of $\Omega \sqrt{K}$ are: (a) $\Omega \sqrt{K}=0.1 \sqrt{N-1}$, (b) $\Omega \sqrt{K}=0.15 \sqrt{N-1}$, (c) $\Omega \sqrt{K}=0.25 \sqrt{N-1}$, and (d) $\Omega \sqrt{K}=\sqrt{N-1}$. The different lines in each pannel correspond to different $K$. Solid (black) line: $K=2$; dashed (red) line: $K=4$; dotted (green) line: $K=6$, and dash-dotted (blue) line: $K=N-1$. Reprinted with permission from [25], S. Yuan et al., Phys. Rev. B 77, 184301 (2008). (C) 2008

if $\Omega \sqrt{K}$ increases (compare Figs. 4(a) to (d)), the differences between the Loschmidt echoes increase.

According to the general picture of decoherence, ${ }^{9}$ for an environment with nontrivial internal dynamics that is initially in a random superposition of all its eigenstates, we expect that the quantum system will evolve into a stable mixture of its eigenstates. In other words, the decoherence will cause all the off-diagonal elements of the reduced density matrix to vanish with time. In the case of an isotropic Heisenberg coupling between the quantum system and the environment, $H_{\mathrm{S}}$ commutes with the Hamiltonian $H$, hence the energy of the quantum system is a conserved quantity. Therefore, the weight of the singlet $|S\rangle$ in the mixed state should be a constant $(1 / 2)$, and the weights of the degenerate eigenstates $\left|T_{0}\right\rangle,\left|T_{-1}\right\rangle$ and $\left|T_{1}\right\rangle$ are expected to become the same (1/6). As shown in Figures 2(b-e)(right), our simulations confirm that this picture is correct in all respects.

\subsection{Decoherence with Energy Dissipation}

Now we consider the case that there is energy dissipation of the quantum system, i.e., $\left[H_{\mathrm{S}}, H\right] \neq 0$. First, instead of considering a Heisenberg system-environment interaction
$H_{\mathrm{SE}}$ as in Figures 2-3, we now take a Heisenberg-type $H_{\mathrm{SE}}$ in Figures 5-6, the other interactions like $H_{\mathrm{S}}$ and $H_{\mathrm{E}}$ are the same as in Figures 2-3. From a direct comparison of these results, it is clear that the roles of $K$ and $\Omega$ are the same, no matter whether the energy of the quantum system is conserved or not. If $|\Delta| \gg \Omega \sqrt{K}$, in the presence of anisotropic interactions between the quantum system and the environment spins, the second step of the oscillations decay and finally disappear as $K$ increases, even in the absence of interactions between the bath spins. This is because the anisotropic interactions break the rotational symmetry of the coupling between the quantum system and the environment which is required for the long-living oscillations to persist. If $|\Delta| \ll \Omega \sqrt{K}$ and $\Omega$ is comparable with $J,\left|\rho_{23}(t)\right|$ can still be described by Eq. (33), but now $A_{\mathrm{K}}(\Omega)$ is no longer a linear function of $\Omega$. This is because the energy dissipation will change the weight of each pointer state (eigenstate) in the final stable mixture, which makes the time evolution of $\left|\rho_{23}(t)\right|$ more complicated.

More results with Ising \pm interaction $H_{\mathrm{SE}}$ are shown in Figures 7 and 8 . They give similar results as in the case of a Heisenberg-type interaction $H_{\mathrm{SE}}$. The environments in Figure 8 are not a spin glass, but an isotropic Heisenberg 

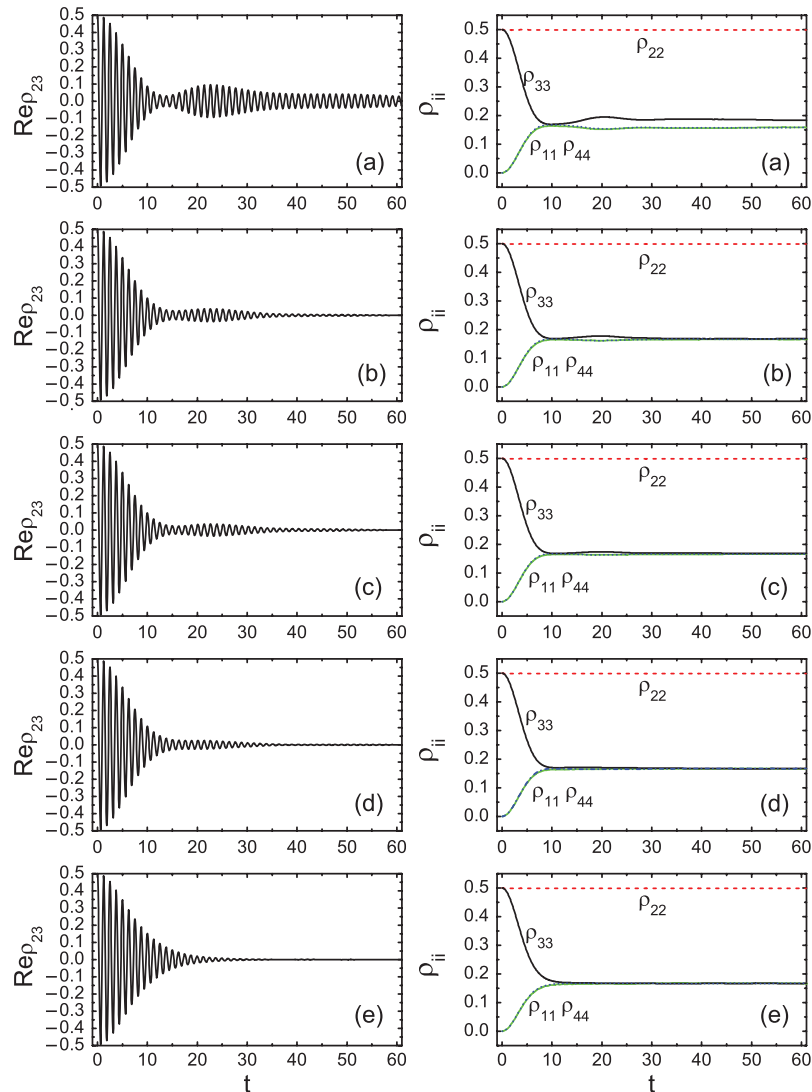

Fig. 5. Time evolution of the real part of the off-diagonal element $\rho_{23}$ (left panel) and the diagonal elements $\rho_{11}, \ldots, \rho_{44}$ (right panel) of the reduced density matrix of a Heisenberg two-spin system $(J=-5)$, coupled via an isotropic Heisenberg-type interaction $H_{\mathrm{SE}}(\Delta=-0.15)$ to a Heisenberg-type environment $H_{\mathrm{E}}(\Omega=0.15)$ with different connectivity: (a) $K=0$; (b) $K=2$; (c) $K=4$; (d) $K=6$; (e) $K=N-1$.

antiferromagnetic square or triangle lattice, which are also frustrated systems.

\subsection{Summary}

In conclusion, with a frustrated spin-bath environment that initially is in a random superposition of its basis states, a pure quantum state of the quantum spin system will evolve into a classical mixed state. If the interaction between the quantum system and environment is much smaller than the coupling between the spins in the quantum system, the pointer states are the eigenstates of the quantum system. Both of these observations are in concert with the general picture of decoherence. ${ }^{9}$ Furthermore, if the energy of the quantum system is conserved, the pointer states can still be the eigenstates of the quantum system, independent of the ratio of the system-bath coupling to the coupling within the system. For the anisotropic spin-bath, changing the internal dynamics of the environment (geometric structure or exchange couplings) may change the decoherence of the quantum spin system from Gaussian to exponential decay.

\section{EVOLUTION TO THE GROUND STATE OF A TWO-SPIN SYSTEM}

To approach the ground state of a quantum system by coupling it to a large quantum bath is not a trivial problem. The interactions between the two quantum systems will not only lead to the exchange of the energy, but also the coherence of the wave function. In this section, we show under which condition a two-spin system can approach its ground or near-ground state. The affect of entanglement and interaction symmetry during this evolution will be discussed.

The interaction within the two-spin system will be fixed as isotropic Heisenberg ferromagnet $(J>0)$ or antiferromagnet $(J<0)$. In the case of ferromagnet, there are three degenerate ground states (triplet states) $\left|T_{0}\right\rangle,\left|T_{1}\right\rangle$ and $\left|T_{-1}\right\rangle$, whereas in the case of antiferromagnet it is the singlet state $|S\rangle$. Therefore when the quantum system approaches the state which has the same energy as the ground state(s), it can be a single eigenstate if the ground state is non-degenerate, and it can also be an entangled superposition (quantum) or mixed state (classic) if the ground states are degenerate.

In order to let the two-spin system approach the ground state, it is necessary to keep the environment at low temperature. Instead of a classical mixture, we will prepare the environment in a pure quantum state, in which the temperature is not well defined. So we simply initialize the environment in its ground or near-ground state to guarantee the one-direction energy flow. As in Refs. [25, 26], this initial ground state of the environment leads to a sharp local density of states in the whole system, and therefore the decoherence of the quantum system is much weaker comparing to cases with random initial state of the environment.

The interactions between the two-spin system and the spin bath will be set as Heisenberg-type or Ising-type. Even in both cases the interactions of different orientations ( $x, y$ and $z$ ) are not all the same, but they are totally different: the Heisenberg-type $H_{\mathrm{SE}}$ is still symmetrical because the exchange interactions have the same random amplitude in the three orientations, whereas the Ising-type $H_{\mathrm{SE}}$ is antisymmetric since the exchange interactions are totally different between $x(y)$ and $z$ directions.

The interactions within the environment are fixed as Heisenberg-type, which reduce the strength of the coherence between the quantum system and the environment, as we showed in the previous section and Refs. [23-26].

\subsection{Symmetrical Coupling}

We first consider the case that an antiferromagnetic $(J<0)$ or ferromagnetic $(J>0)$ quantum system that interacts with the Heisenberg-type environment via a Heisenbergtype interaction.

In Figure 9, we present simulation results for the twospin correlation function (as a measure of the energy) 

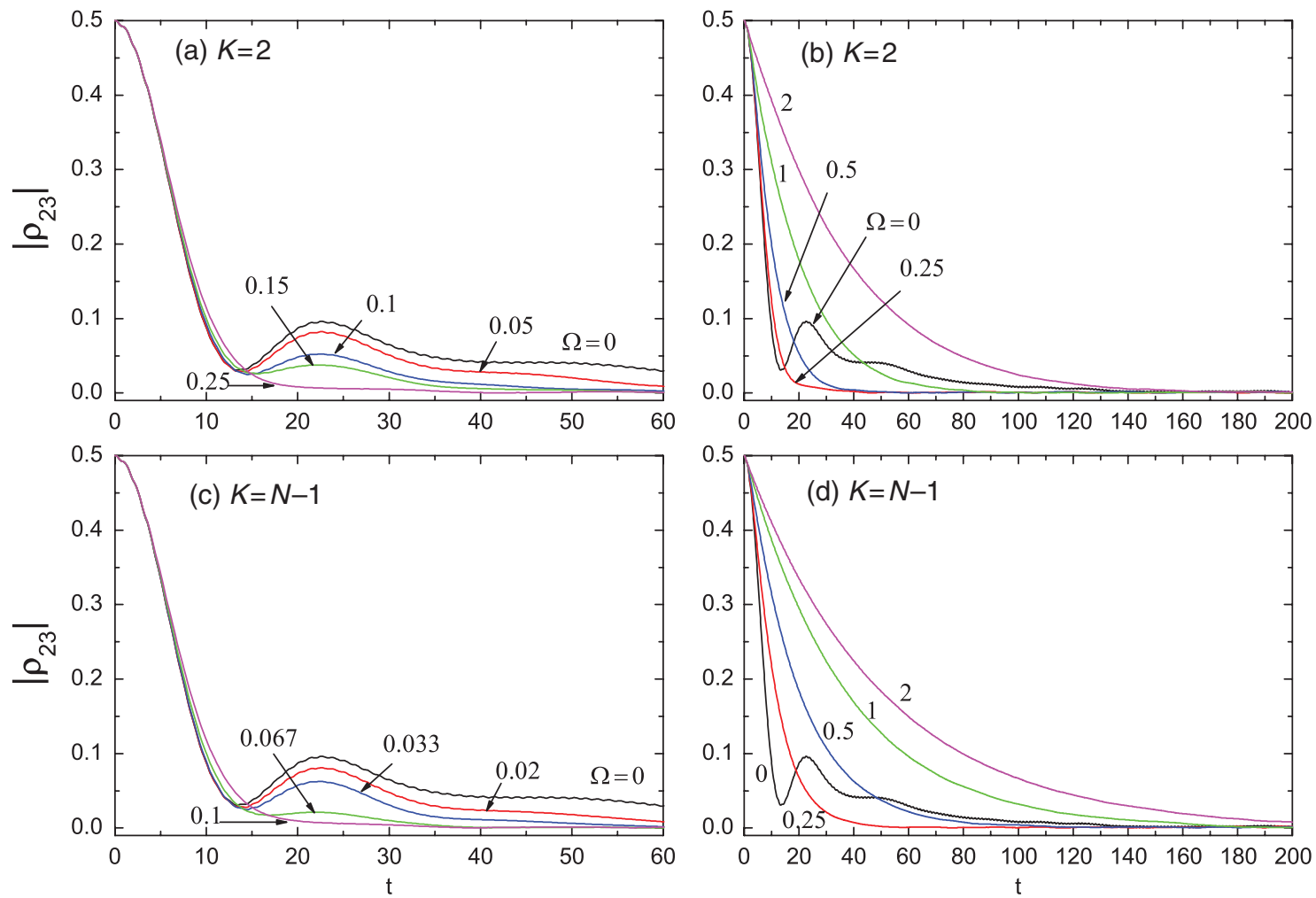

Fig. 6. Same as Figure 3 except that $H_{\mathrm{SE}}$ is Heisenberg-type and $\Delta=0.15$. Reprinted with permission from [25], S. Yuan et al., Phys. Rev. B 77, 184301 (2008). (C) 2008.

for different values of the coupling strength $(\Omega)$ in the environment. Clearly, in case (a), the relaxation in both cases, antiferromagnetic and ferromagnetic, is rather slow and confirming that there is relaxation to the ground state requires a prohibitively long simulation. For cases (b)-(d), the results are in concert with the intuitive picture of relaxation due to decoherence: The correlation shows the relaxation from the up-down initial state of the quantum system to the ground or near-ground state. As for the antiferromagnetic quantum system $(J<0)$, the two-spin correlation relaxes to a value of about $0.65-0.70$, which is much further away from the ground state value $-3 / 4$ than we would have expected on the basis of the results of the ferromagnetic quantum system. In the true ground state of the whole system, the value of the two-spin correlation in case (b) of $J<0$ is -0.7232 , and hence significantly lower than the typical values, reached after relaxation. On the one hand, it is clear (and to be expected) that the coupling to the environment changes the ground state of the quantum system, but on the other hand, our numerical calculations show that this change is too little to explain the apparent difference from the results obtained from the time-dependent solution. In case (e), the characteristic strength of the interactions between the spins in the environment is of the same order as the exchange coupling in the quantum system $(\Omega \approx J)$, a regime in which there clearly is significant transfer of energy, back-and-forth, between the quantum system and the environment.
In Figure 10, we show the diagonal elements of the reduced density matrix for case (b). After reaching the steady state, the nondiagonal elements exhibit minimum fluctuations about zero and are therefore not shown. From Figure 10, it is then clear that the quantum system relaxes to the singlet state for the antiferromagnetic $(J<0)$ system, and to a mixed state for the ferromagnetic system $(J>0)$, as expected on intuitive grounds.

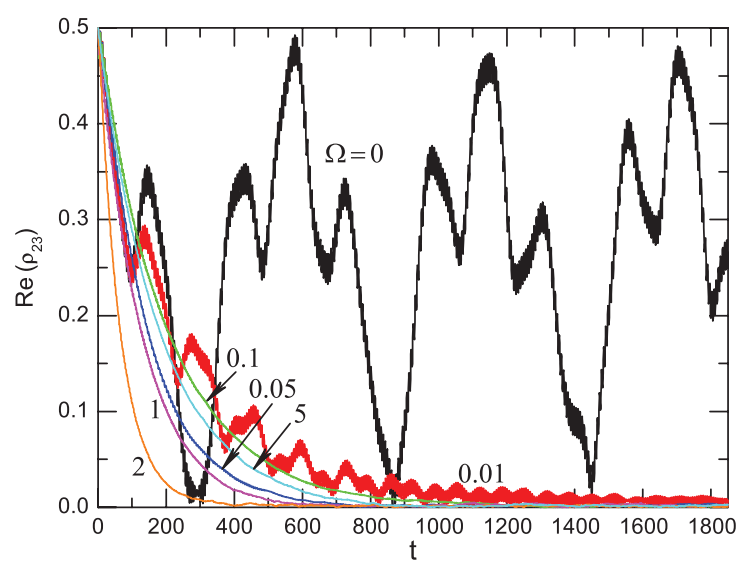

Fig. 7. Time evolution of the real part of the off-diagonal element $\rho_{23}$ of the reduced density matrix of a Heisenberg two-spin system $(J=-5)$, coupled via an Ising \pm interaction $H_{\mathrm{SE}}(\Delta=0.075)$ to a Heisenberg-type-ring environment $H_{\mathrm{E}}(N=16)$ with different range of $\Omega$ $(0,0.01,0.05,0.1,1,2,5)$. The value of the corresponding $\Omega$ is indicated by the number near each line. 
An important observation is that our data convincingly shows that it is not necessary to have a macroscopically large environment for decoherence to cause relaxation to the ground state: A spin-glass with $N=14$ spins seems to be more than enough to mimic such an environment for a two-spin system. This observation is essential for numerical simulations of relatively small systems to yield the correct qualitative behavior.

Qualitative arguments for the high efficiency of the spinglass bath were given in Ref. [23]. Since spin-glasses possess a huge amount of states that have an energy close to the ground state energy but have wave functions that are very different from the ground state, the orthogonality catastrophe, blocking the quantum interference in the quantum system ${ }^{3,9}$ is very strongly pronounced in this case.

From the data for (b)-(d), shown in Figure 9, we conclude that the time required to let the quantum system relax to a state that is close to the ground state depends on the energy scale $(\Omega)$ of the random interactions between the spins in the environment. As it is difficult to define the point in time at which the quantum system has reached its stationary state, we have not made an attempt to characterize the dependence of the relaxation time on $\Omega$.

\subsection{Antisymmetric Coupling}

In our simulation, the initial state of the quantum system is $|\uparrow \downarrow\rangle$ and this state has total magnetization $M=0$. For an Ising-type interaction $H_{\mathrm{SE}}$ of the envionment with a Heisenberg system $H_{\mathrm{S}}$, the magnetization $M$ of the quantum system commutes with the Hamiltonian of the whole system.
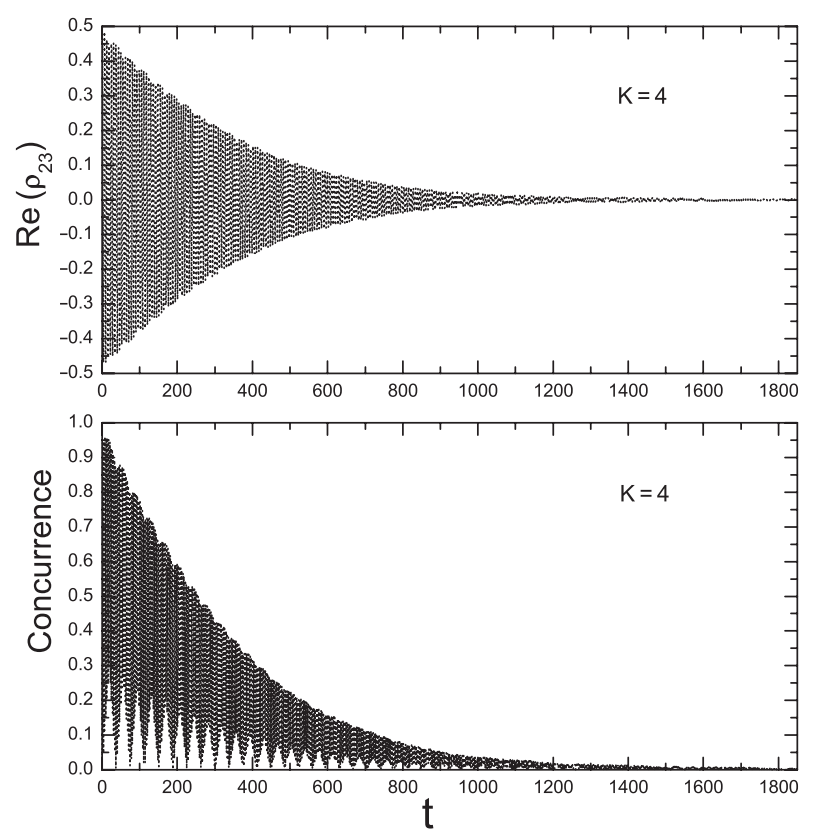

Therefore, the magnetization of the quantum system is conserved during the time evolution, and the quantum system will always stay in the subspace with $M=0$. In this subspace, the ground state for the antiferromagnetic quantum system is the singlet state $|S\rangle$ while for the ferromagnetic quantum system the ground state (in the $M=0$ subspace) is the entangled state $\left|T_{0}\right\rangle$. Thus, for the Ising-type interaction $H_{\mathrm{SE}}$, starting from the initial state $|\uparrow \downarrow\rangle$, the quantum system should relax to an entangled state, for both a ferroor antiferromagnetic quantum system, that is, at any time $t$, the state of the whole system can be written as

$$
|\Psi(t)\rangle=|S\rangle\left|\Phi_{\mathrm{S}}(t)\right\rangle+\left|T_{0}\right\rangle\left|\Phi_{T_{0}}(t)\right\rangle
$$

where $\left|\Phi_{\mathrm{S}}\right\rangle$ and $\left|\Phi_{T_{0}}\right\rangle$ denote the states of the environment.

Let us denote by $\left\{\left|\phi_{i}\right\rangle\right\}$ a complete set of states of the environment. Within the subspace spanned by the states $\left\{|S\rangle\left|\phi_{i}\right\rangle,\left|T_{0}\right\rangle\left|\phi_{i}\right\rangle\right\}$, the Hamiltonian can be written as

$$
\begin{aligned}
H= & E_{\mathrm{S}}|S\rangle\left\langle S\left|+E_{\mathrm{T}}\right| T_{0}\right\rangle\left\langle T_{0}\right|+H_{\mathrm{E}} \\
& -\frac{1}{2} \sum_{j=1}^{N}\left(\Delta_{1, j}^{(z)}-\Delta_{2, j}^{(z)}\right)\left(|S\rangle\left\langle T_{0}|+| T_{0}\right\rangle\langle S|\right) I_{j}^{z}
\end{aligned}
$$

where we used $\left\langle S\left|S_{1}^{z}\right| S\right\rangle=\left\langle T_{0}\left|S_{1}^{z}\right| T_{0}\right\rangle=\left\langle S\left|S_{2}^{z}\right| S\right\rangle=$ $\left\langle T_{0}\left|S_{2}^{z}\right| T_{0}\right\rangle=0,\left\langle T_{0}\left|S_{1}^{z}\right| S\right\rangle=1 / 2$, and $\left\langle T_{0}\left|S_{2}^{z}\right| S\right\rangle=-1 / 2$.

Introducing a pseudo-spin $\sigma=\left(\sigma^{x}, \sigma^{y}, \sigma^{z}\right)$ such that the eigenvalues +1 and -1 of $\sigma^{z}$ correspond to the states $|S\rangle$ and $\left|T_{0}\right\rangle$, respectively, Eq. (36) can be written as

$$
\begin{aligned}
H= & \frac{E_{\mathrm{S}}-E_{\mathrm{T}}}{2}+\frac{E_{\mathrm{S}}+E_{\mathrm{T}}}{2} \sigma^{\mathrm{z}}+H_{\mathrm{E}} \\
& -\frac{1}{2} \sum_{j=1}^{N}\left(\Delta_{1, j}^{(z)}-\Delta_{2, j}^{(z)}\right) I_{j}^{z} \sigma^{x}
\end{aligned}
$$
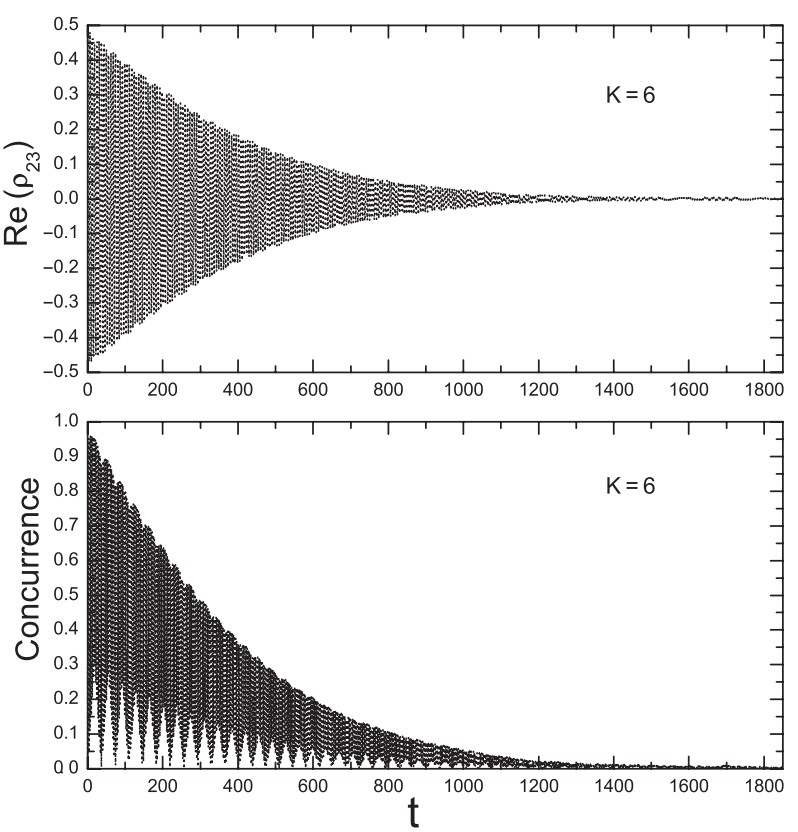

Fig. 8. Time evolution of the real part of the off-diagonal element $\rho_{23}$ and concurrence of the reduced density matrix of a Heisenberg two-spin system $(J=-1)$, coupled via an Ising interaction $\pm H_{\mathrm{SE}}(\Delta=0.075)$ to an isotropic Heisenberg environment $H_{\mathrm{E}}(\Omega=-0.15, N=16)$ with connectivity $K=4$ (square lattice) and $K=6$ (triangle lattice). 

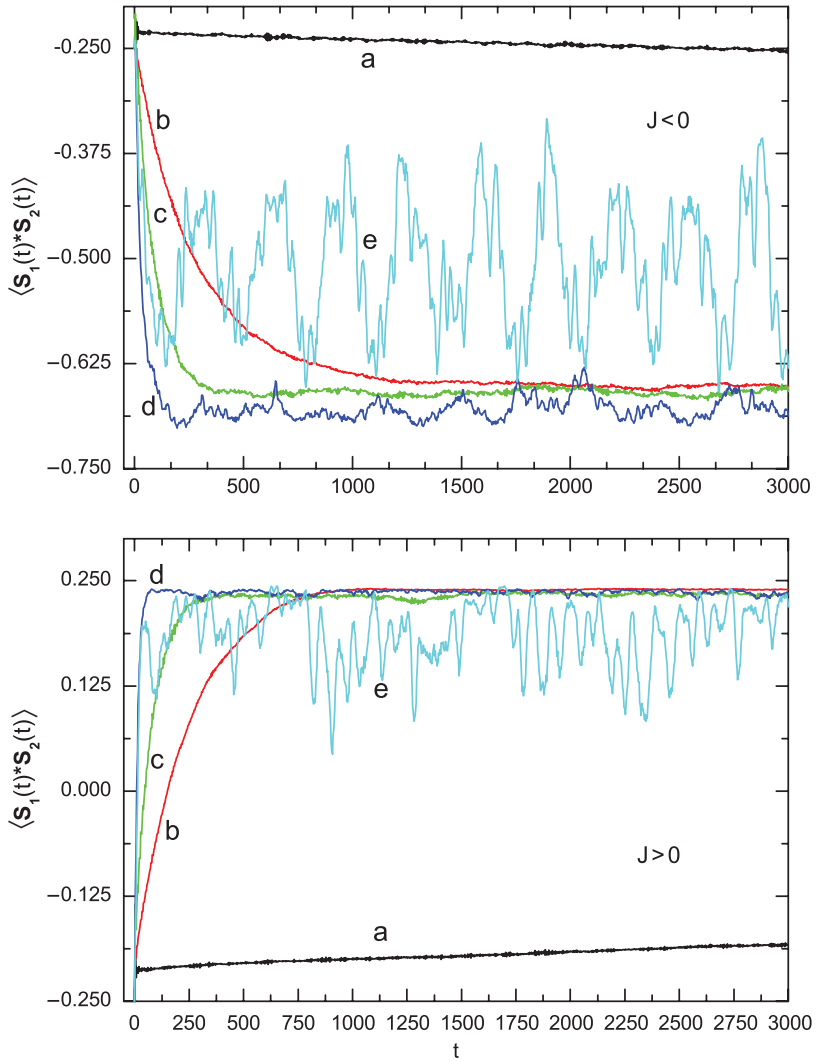

Fig. 9. Time evolution of the correlation $\left\langle\Psi(t)\left|\mathbf{S}_{1} \cdot \mathbf{S}_{2}\right| \Psi(t)\right\rangle$ of the antiferromagnetic (top panel) and ferromagnetic (bottom panel) two-spin quantum system with Heisenberg-type interaction $H_{\mathrm{SE}}$ and environment $H_{\mathrm{E}}$. The model parameters are $\Delta=0.15$ and (a) $\Omega=0.075$; (b) $\Omega=0.15$; (c) $\Omega=0.20$; (d) $\Omega=0.30$; (e) $\Omega=1$. The number of spins in the environment is $N=14$.

showing that in the case of Ising-type $H_{\mathrm{SE}}$, the quantum system with two spins is equivalent to the model Eq. (37) with one spin.

From Eq. (37), it follows immediately that the Hamiltonian is invariant under the transformation $\left\{J, \sigma^{z}\right\} \rightarrow$ $\left\{-J,-\sigma^{z}\right\}$. Indeed, the first, constant term in Eq. (37) is irrelevant and we can change the sign of the second term by rotating the speudo-spin by 180 degrees about the $x$-axis. Therefore, if the initial state is also invariant under this transformation, the time-dependent physical properties will not depend on the choice of the sign of $J$, hence the ferro- and antiferromagnetic system will behave in exactly the same manner. In our case, the initial state can be written as $\left(|S\rangle+\left|T_{0}\right\rangle\right)\left|\phi_{0}\right\rangle / \sqrt{2}$, which is trivially invariant under the transformation $\sigma^{z} \rightarrow-\sigma^{z}$.

Therefore for Ising-type $H_{\mathrm{SE}}\left(\Delta_{i, j}^{(x)}=\Delta_{i, j}^{(y)}=0\right)$, an initial state that is invariant for the transformation $|S\rangle \leftrightarrow\left|T_{0}\right\rangle$ ), $\langle\Psi(t)|A| \Psi(t)\rangle$ does not depend on the sign of $J$, for any observable $A$ of the quantum system that is invariant for this transformation. Under these conditions, it is easy to prove that

$$
\left\langle\Psi(t)\left|\mathbf{S}_{1} \cdot \mathbf{S}_{2}\right| \Psi(t)\right\rangle_{F}+\left\langle\Psi(t)\left|\mathbf{S}_{1} \cdot \mathbf{S}_{2}\right| \Psi(t)\right\rangle_{A}=-\frac{1}{2}
$$
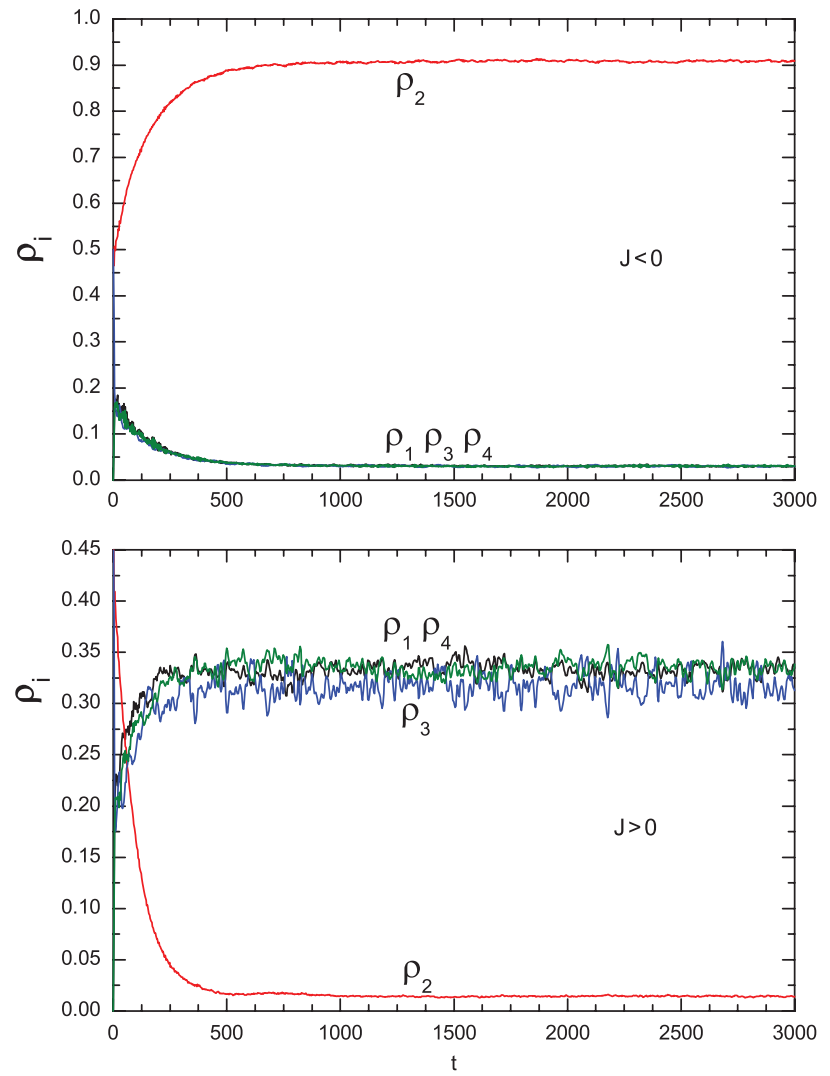

Fig. 10. Time evolution of the diagonal matrix elements of the reduced density matrix of the antiferromagnetic (top panel) and ferromagnetic (bottom panel) two-spin quantum system for $\Delta=0.15$ and $\Omega=0.15$ (case (b) of Figure 9, except that the number of spins in the environment is $N=16$ ).

where the subscript $F$ and $A$ refer to the ferro- and antiferromagnetic quantum system, respectively.

Likewise, for the concurrence we find $C_{\mathrm{F}}(t)=C_{A}(t)$ and similar symmetry relations hold for the other quantities of interest. Of course, this symmetry is reflected in our numerical data also, hence we can limit ourselves to present data for the antiferromagnetic quantum system with Ising-type $H_{\mathrm{SE}}$.

In Figures 11 and 12, we present simulation results for the two-spin correlation function and concurrence for different values of the parameter $\Delta$ and $\Omega$. It is clear that for a certain range of the interaction strength, the quantum system relaxes to a state that is very close to the ground state, see Figures 11(c) and 12(c). That is, the presence of a conserved quantity (the magnetization of the quantum system) acts as a catalyzer for relaxing to the ground state. Intuitively, we would expect that the presence of a conserved quantity hinders the relaxation and indeed, the relaxation in Figure 11 is much slower than in Figure 9. Notwithstanding this, in the presence of a conserved quantity, the quantum system relaxes to a state that is much closer to the true ground state than the one it would relax to in the absence of this conserved quantity. 


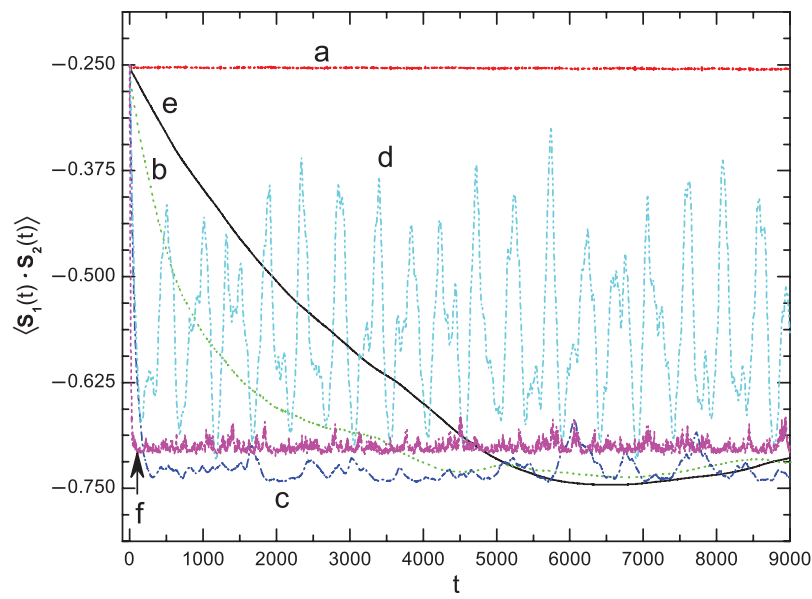

Fig. 11. Time evolution of the correlation $\left\langle\Psi(t)\left|\mathbf{S}_{1} \cdot \mathbf{S}_{2}\right| \Psi(t)\right\rangle$ of the antiferromagnetic quantum system with Ising-type $H_{\mathrm{SE}}$ and Heisenbergtype $H_{\mathrm{E}}$. The model parameters are (a-d) $\Delta=0.075$, (e) $\Delta=0.0375$, (f) $\Delta=0.15$, and (a) $\Omega=0.075$, (b,e) $\Omega=0.15$, (c, f) $\Omega=0.30$, (d) $\Omega=1$. The number of spins in the environment is $N=16$.

One should notice that only in a small range of parameters $\Delta$ and $\Omega$ the two-spin quantum system can evolve into a near-ground state. If the interactions within the bath is too small, the range of the energy spectrum of the bath limits the energy dissipation of the system, and the system
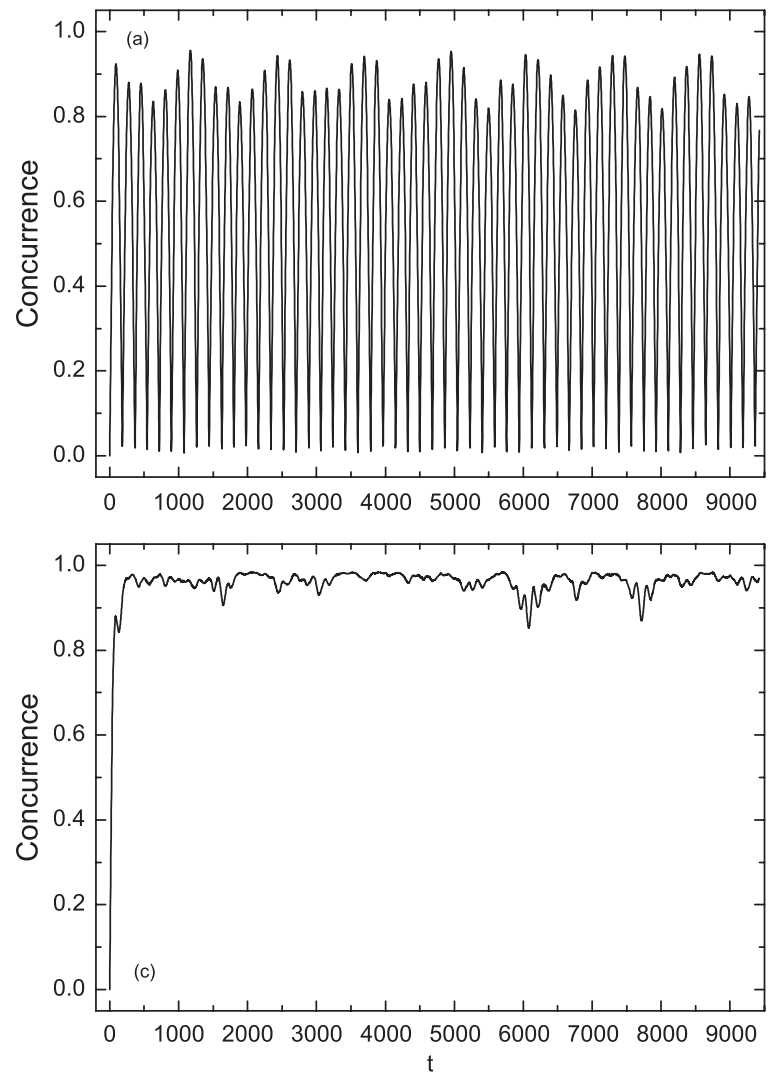

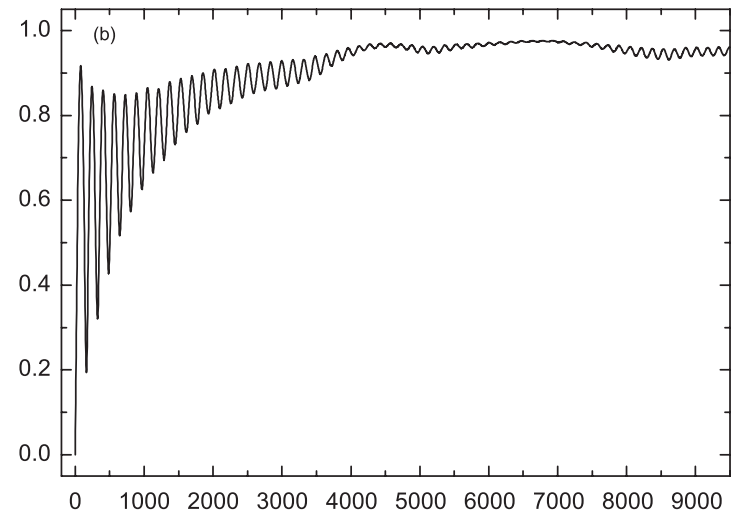

spins mainly follow its own dynamics just like there is no bath. On the contrary, if the interactions within the bath are too strong, a small change of the configuration of the bath spins will lead to a large change of its energy, therefore the direction of the energy flow will oscillate with time and the quantum system cannot arrive to a state with approximately stable energy.

\subsection{Summary}

In general, it turns out that the relaxation to the ground state is a more complicated process than one would naively expect, depending essentially on the ratio between parameters of the interaction and environment Hamiltonians. Two general conclusions are:

(1) the quantum system more easily evolves into its ground state when the latter is more degenerate (degenerate triplet states compared to the singlet) or less entangled (e.g., up-down state compared to the singlet);

(2) constraints on the system such as existence of additional integrals of motion can make the evolution to the ground state more efficient.

An explanation of the first statement is that if the ground state is more degenerate, its affective dimension in the Hilbert space is larger and therefore the wave function

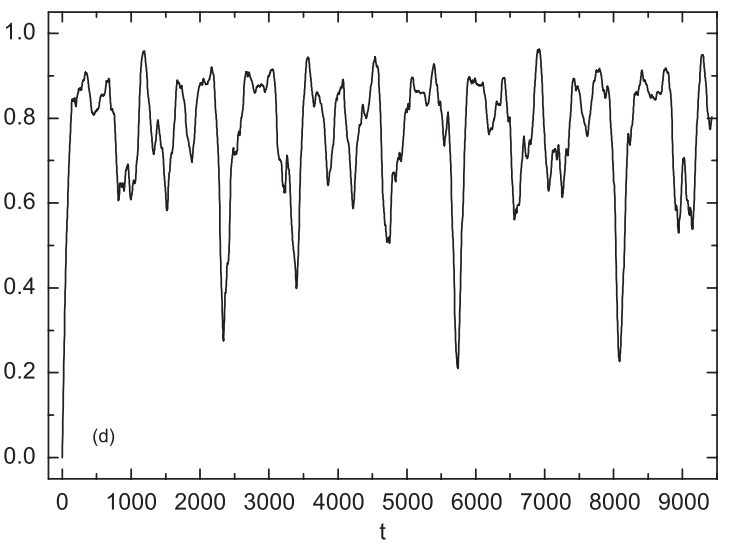

Fig. 12. Time evolution of the concurrence of the antiferromagnetic quantum system with Ising-type interaction $H_{\mathrm{SE}}$ and Heisenberg-type environment $H_{\mathrm{E}}$. The model parameters are $\Delta=0.075$ and (a) $\Omega=0.075$, (b) $\Omega=0.15$, (c) $\Omega=0.30$, (d) $\Omega=1$. The number of spins in the environment is $N=16$. 
will have more possibilities to evolve into this subspace, especially if the environment is frustrated. The latter statement looks a bit counterintuitive since it means that it may happen that a more regular system exhibits stronger relaxation than a chaotic one. The reason that it may happen is that introducing an additional integral of motion, such as the total magnetization, limits the dimensionality of the available Hilbert space for the quantum system. The larger the dimensionality of the available Hilbert space, the more complicated the decoherence process is due to the appearance of the whole hierarchy of decoherence. A manifestation of this phenomenon has been observed earlier: ${ }^{20}$ Under certain conditions, the same quantum system as studied here (four by four reduced density matrix) displays "quantum oscillations without quantum coherence" whereas for a single spin in a magnetic field (two by two reduced density matrix) decoherence can, relatively easily, suppress the Rabi oscillations completely.

\section{DECOHERENCE OF A MANY-SPIN SYSTEM}

In the previous sections, we focused on the quantum system with only two spins. Starting from this section we will consider more complicated systems which contain more spins, e.g., four to eight spins.

In general when a quantum system interacts with a quantum environment, there will be energy dissipation and entanglement of their wave functions. This entanglement does not necessary lead to a classical mixed state of the quantum system, especially if the environment has finite size. But introducing certain properties, a finite quantum environment can also drive the quantum system to an exact classical state. In the previous sections, we showed that a frustrated environment can enhance the decoherence of a two-spin system, and as we will see in what follows, this is also the case for the many-spin system.

\subsection{Origin of the Microcanonical Distribution}

Let's first consider the case that there is no energy dissipation or the energy dissipation is very small. In this case the decoherence of the quantum system fully originates from the phase correlation of the environment. In Figure 13, a Heisenberg-ring with four spins is coupled to a frustrated spin glass consisting of 14 spins, and the interaction between the quantum system and the environment is isotropic or anisotropic but very small (comparing to the coupling within the system, i.e., $\Delta=0.15 \ll|J|=5$ ). In the former case the energy of the quantum system is conserved, and in the latter case the energy dissipation is very small so that it can be ignored. The system has four distinct eigenvalues $\left(E_{1}=-2, E_{2-4}=-1, E_{5-11}=0\right.$, and $\left.E_{12-16}=1\right)$ and sixteen different eigenstates. During the time-integration of the TDSE, the reduced density matrix of the system is calculated every $\tau=\pi / 10$. In both cases, the diagonal terms of the reduced density matrix of the Heisenberg-ring approach a stable value after an initial decay or increase, and the off-diagonal terms are all zero $(\sigma \rightarrow 0)$, which means that in both cases, the four spin quantum system approaches a fully decoherent state.

Another significant result is that the degenerated energy eigenstates have the same weigth distribution in the fully decoherent state, i.e., $\gamma \rightarrow 0$, indicating that the final system is a microcanonical state in each eigenenergy subspace. The diagonal elements of the reduced density matrix corresponding to degenerate eigenstates with zero weight in the initial state are zero and remain so during the time revolution, see $\rho_{5-11}$ in Figure 13. This is simply due to the conservation of energy of the system, and due to the
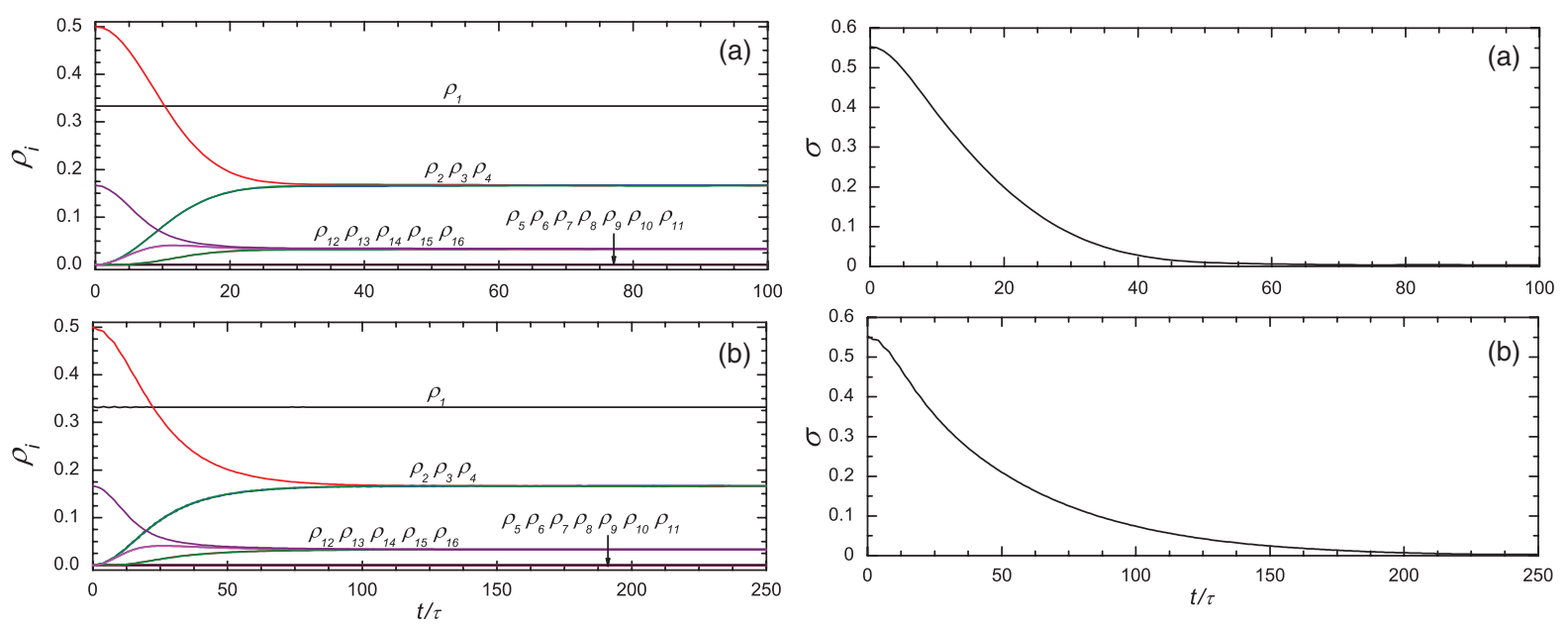

Fig. 13. Time evolution of the diagonal terms $\left(\rho_{i}\right)$ and sum of the absolute values of the off-diagonal terms $(\sigma)$ in the reduced density matrix of a Heisenberg-ring $H_{\mathrm{S}}\left(J=-5, n_{\mathrm{S}}=4\right.$, initial state $\left.|U D\rangle_{\mathrm{S}}\right)$ coupled to a spin glass environment $H_{\mathrm{E}}\left(\Omega=0.15, n=16\right.$, initial state $\left.|R A N D O M\rangle_{\mathrm{E}}\right)$ via (a) Heisenberg interaction $H_{\mathrm{SE}}(\Delta=0.075)$ or (b) Heisenberg-type interaction $H_{\mathrm{SE}}(\Delta=0.15)$. Full decoherence is observed in both cases, and the system $S$ relaxes to a state with equal weights within each energy subspace, that is, $\gamma \rightarrow 0$, a microcanonical ensemble. 


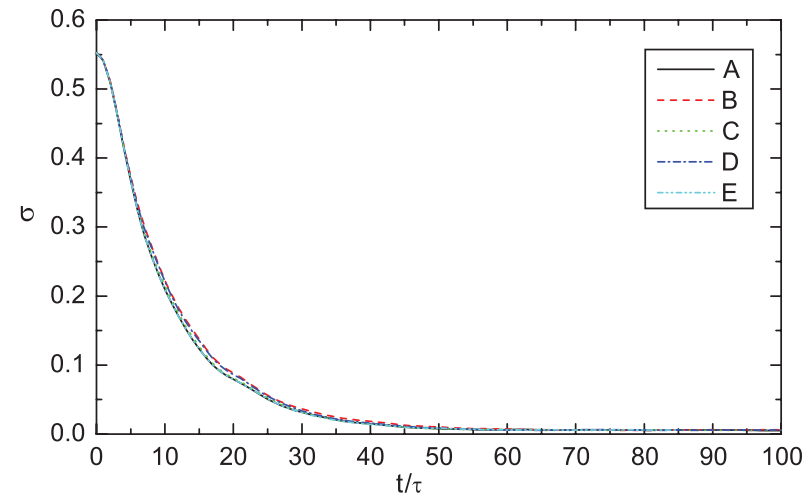

Fig. 14. The system represented here is the same as the one in Figure 13(a), except that the randomness in the coupling constants of $H_{\mathrm{SE}}$ or $H_{\mathrm{E}}$, or the randomness in the initial state of the environment, are different in the curves $A-E$. It is clear that the time evolution of $\sigma(t)$ is not sensitive to the different random values of the coupling constants or the initial state of the environment if they follow the same type of random distribution.

fact that the time evolution operator $e^{-i H t}$ prevents changing the coefficients corresponding to these eigenstates.

In Figure 14, we plot the time evolution of $\sigma(t)$ with different random realizations for the initial state of the environment or for the model parameters $\Omega_{i, j}^{\alpha}$ and $\Delta_{i, j}^{\alpha}$. The difference between the curves is very small, indicating that in our model, a particular randomness of the coupling parameters or initial state is not relevant to the general properties of the simulation results.

In Figure 15, we show the time evolution of $\sigma(t)$ for different coupling strengths $(\Omega)$ in the environment. In general, increasing the coupling strength within the environment will increase the effective energy range of the bath, which leads the decoherence more completely. But as we have shown in the case of the two-spin system, the coupling strength should not be too large, otherwise the energy resolution of the bath will be too small to lead the full decoherence of the system.

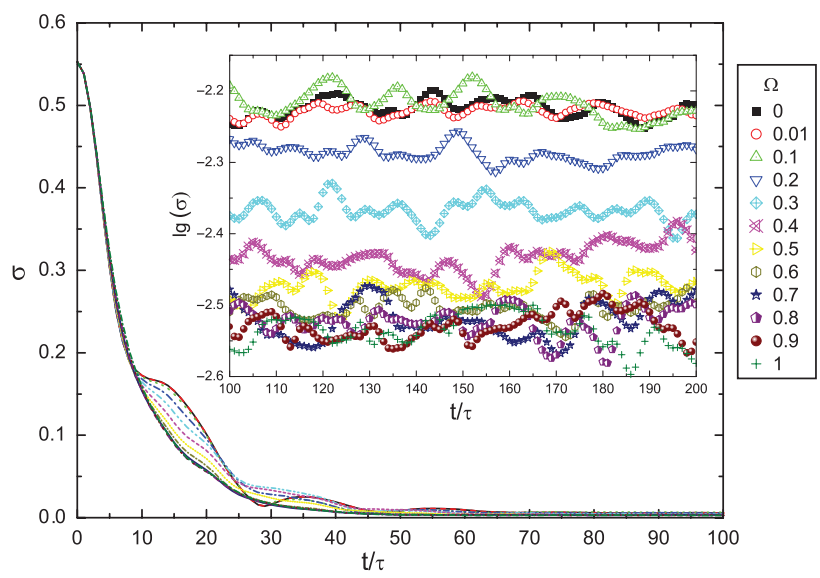

Fig. 15. Same as Figure 13(a) except that the range of the coupling strength $(\Omega)$ in the environment is different.

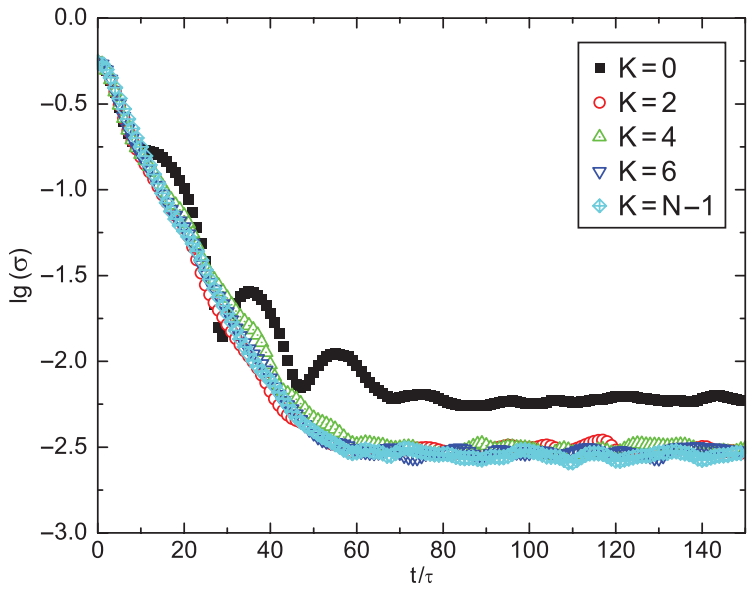

Fig. 16. Same as Figure 13(a) except that the topological structure (connectivity $K$ ) of the environment is different.

In Figure 16, we show the time evolution of $\lg [\sigma(t)]$ for the same systems but with different topological structures (connectivity $K$ ) in the environment. It is clear that as soon as there is frustrated interaction within the environment $(K>0)$, no matter what kind of topological structure it is, the decoherence of the quantum system is quite similar.

\subsection{Summary}

If there is no energy dissipation, or the energy dissipation is so small that it can be ignored, then the entanglement between the quantum system and the environment occurs only in the subspace of the (degenerate) eigenstates which have nonzero weigth distribution in the initial state of the quantum system. That is, the possible pointer states in the mixed states are determined by the initial state of the quantum system itself. If a particular environment can lead to the decoherence of the quantum system without energy dissipation, then turning on the energy dissipation will still lead to decoherence, and even more completely. In fact, energy dissipation is not related to the question whether a quantum system can evolve into a classical mixed state or not. The main difference between decoherence with or without energy dissipation are the number of possible pointer states in the mixed state. That is, an additional integral of motion of the system will limit the number of pointer states, and therefore a full decoherence state is a mixture with microcanonical distribution in each eigenenergy subspace under the extra conservation law. On the contrary, if there is enough energy dissipation between the two systems, then all eigenstates of the quantum system are possible pointer states. And more importantly, as we will show in the next section, the mixed state of the quantum system follows the canonical distribution. ${ }^{26}$ 


\section{THERMALIZATION OF A MANY-SPIN SYSTEM}

In the previous section, we have shown that turning on the interaction between the many-spin system and the environment, leads to a reduction of the coherence in the quantum system. The coupling with the environment causes the initial pure state of the quantum system to evolve into a mixed state, obtained by tracing out all the degrees of freedom of the environment. The pointer states in the mixed state are determined by the initial state of the quantum system if there is an additional integral of motion, e.g., the conserved energy or magnetization. This leads to a microcanonical ensemble under a certain conservation law. On the other hand, if there is enough energy dissipation without any additional integral of motion, we expect that the mixed state is a canonical ensemble. ${ }^{26}$

Earlier demonstrations of the fact that the system can be in the canonical ensemble state are based on Ergodic averages $^{43-46}$ or canonical typicality ${ }^{47-53}$ The Ergodic averages consider the dynamics of a closed quantum system, and prove that in certain quantum systems, the expectation
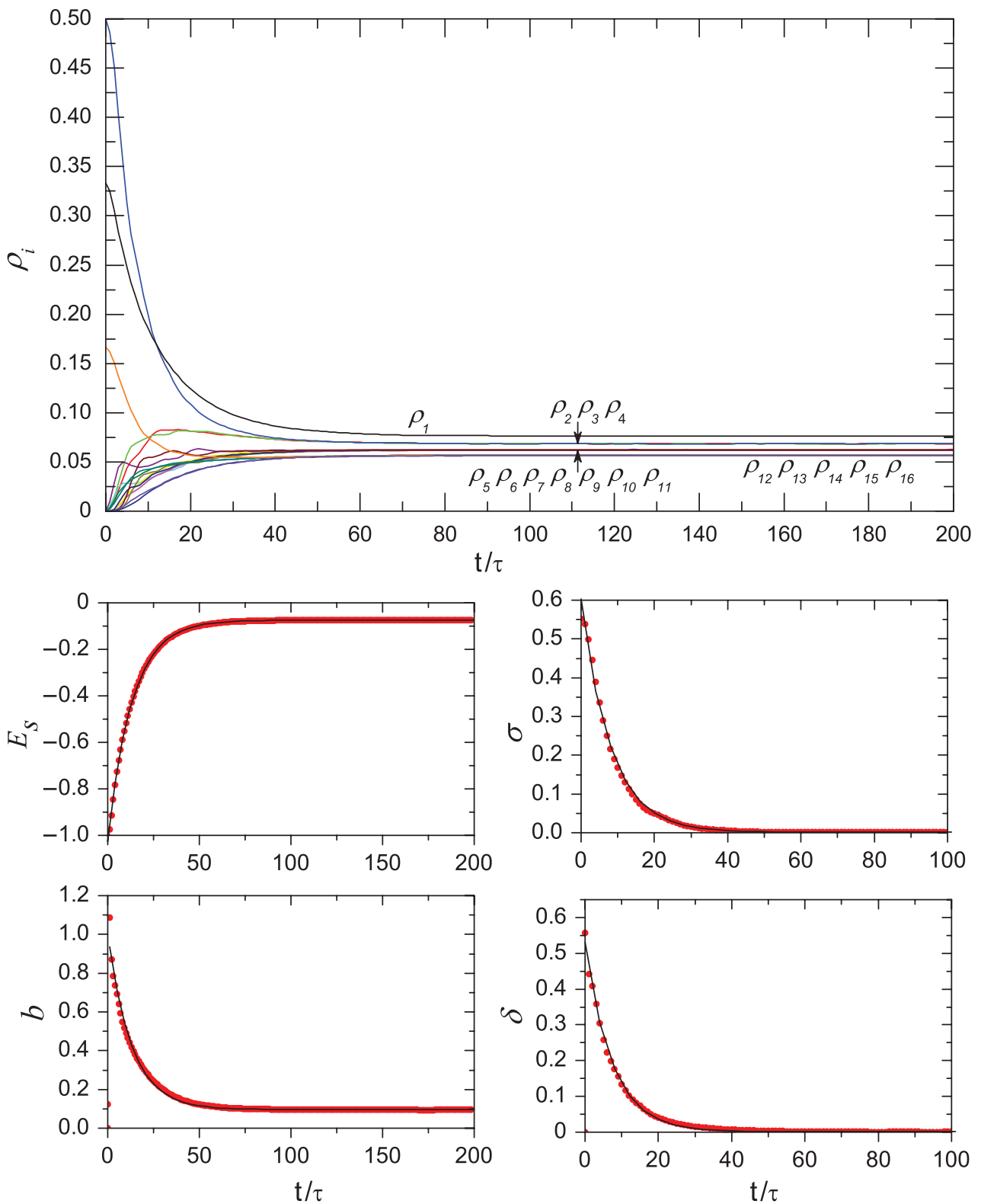

Fig. 17. Simulation results for the diagonal elements $\rho_{i} \equiv \widehat{\rho}_{i i}(t)$ of the density matrix of $S$, the energy $E_{\mathrm{S}} \equiv E_{\mathrm{S}}(t)$, the effective inverse temperature $b \equiv b(t)$ and its variance $\delta \equiv \delta(t)$, and $\sigma \equiv \sigma(t)$ which is a measure for the decoherence in $S$, as obtained by solving the TDSE for the whole system with a Heisenberg-ring $H_{\mathrm{S}}\left(J=-1, n_{\mathrm{S}}=4\right)$, a Heisenberg-type interaction $H_{\mathrm{SE}}(\Delta=0.3)$, a spin glass environment $H_{\mathrm{E}}(\Omega=1, n=18)$, and $\tau=\pi / 10$. The initial state of the whole system is a product state $|U D\rangle_{\mathrm{S}} \otimes|R A N D O M\rangle_{\mathrm{E}}$. The red dots in the small panels represent the simulation data, and the black curves are fitting curves (see text). 
values of the dynamical variables of the system approach their values for the subsystem that is in the thermal equilibrium state. This is similar to the assumption of classical statistical physics, that is, during large enough time, the trajectory of the many particle system in the phase space will pass all possible points, and therefore the average of these points in the phase space will follow a certain distribution. On the contrary, the canonical typicality does not consider the dynamics of the system but assumes that if the whole system is in the microcanonical ensemble then its subsystems are in the canonical ensemble. The statement of the canonical typicality is quite general, but it is clear that the dynamical procedure is missing. Moreover, the coupling between the quantum system and the environment is assumed to be so small that it can be neglected in the theory. Therefore it is important to show how the canonical distribution of statistical mechanics relates to the dynamical evolution of the quantum system.

\subsection{Origin of the Canonical Ensemble}

As a frustrated environment is very effective for creating full decoherence $(\sigma \rightarrow 0)$ in a quantum spin system, and full decoherence is a necessary condition for the state of the system to converge to its canonical distribution, we have chosen spin glass environments, which have no obvious symmetries. ${ }^{26}$

First, we consider a system (Heisenberg-ring $H_{\mathrm{S}}$ ) interacting (Heisenberg-type $H_{\mathrm{SE}}$ ) with an environment (spin glass $\left.H_{\mathrm{E}}\right)$. The system has four distinct eigenvalues $\left(E_{1}=\right.$ $-2, E_{2-4}=-1, E_{5-11}=0$, and $\left.E_{12-16}=1\right)$ and sixteen different eigenstates. The environment has $2^{18}$ eigenstates. During the time-integration of the TDSE, the reduced density matrix of the system is calculated every $\tau=\pi / 10$ as in the previous section As we described earlier, the values of the diagonal elements $\widehat{\rho}_{i i}$ yield an estimate for the effective inverse temperature $b(t)$, the error $\delta(t)$ for this estimate and the measure $\sigma(t)$ for the deviation from a non-diagonal matrix. The energy of the system is obtained as $E_{\mathrm{S}}(t)=\operatorname{Tr}_{\mathrm{S}} \widehat{\rho}(t) H_{\mathrm{S}}$.

From the simulation results, shown in Figure 17, it is clear that for $t>50 \tau$, each diagonal element $\widehat{\rho}_{i i}$ of the reduced density matrix converges to one out of four stationary values, corresponding to the four non-degenerate energy levels of the system. This convergence is a two-step process. First the system looses all coherence, as indicated by the vanishing of $\sigma(t)$ for $t>50 \tau$. The time dependence of $\sigma(t)$ fits very well to an exponential law

$$
\sigma(t)=\sigma_{\infty}+A e^{-t / T_{2}}
$$

with $\sigma_{\infty}=0.00128, A=0.602$ and $T_{2}=8.01 \tau$. In the small panels of Figure 17, the red dots are the simulation data and the black curves are the fitting function. Likewise, the vanishing of $\delta(t)$ on the same time-scale
$\left(T_{2}=7.32 \tau\right)$, indicates that the density matrix of the system converges to the canonical distribution with the same speed of decoherence.

The effective temperature $b(t)$ and the energy of the system $E_{\mathrm{S}}(t)$ also fit very well to the exponential laws

$$
b(t)=\beta+B e^{-t / T_{1}}
$$

and

$$
E(t)=E_{\infty}+C e^{-t / T_{1}}
$$

with $\beta=0.0962, B=-0.900$, and $T_{1}=13.3 \tau$ and $E_{\infty}=$ $-0.0745, C=-0.952$. The estimated values for $T_{1}$ and $T_{2}$ change very little if we choose different random realizations for the initial state of the environment or for the model parameters $\Omega_{i, j}^{\alpha}$ and $\Delta_{i, j}^{\alpha}$ (see Fig. 18). If we change their range, $T_{1}$ and $T_{2}$ also change, as naively expected.

In order to verify the role of the dynamics within the bath to the thermalization of the quantum system, we plot the time evolution of $\delta(t)$ for different coupling strength $(\Omega)$ in Figure 19. Similar as the dependence of $\sigma$ on $\Omega$ in Figure 15, increasing the coupling strength (within a certain range) will increase the effective energy range of the bath, which leads to a more complete decoherence and thermalization.

The simulation results of a similar system with one extra spin in the quantum system $\left(n_{\mathrm{s}}=5\right)$ and one spin less in the environment $(n=17)$ are shown in Figure 21, and are very similar to the ones shown in Figure 17. These simulations demonstrate that the system first looses all coherence and then, on a longer time-scale, relaxes to its thermal equilibrium state with a finite temperature. In terms of the theory of magnetic resonance, ${ }^{96} T_{1}$ and $T_{2}$ are the times of dissipation and dephasing, respectively. In the case of very small $H_{\mathrm{E}}$, one should expect, instead of an exponential decay of $\sigma$ and $E$, a Gaussian decay, as observed in Refs. [23-25].

It is necessary to extend the types of interaction (Hamiltonian) to verify the generality of the above results.

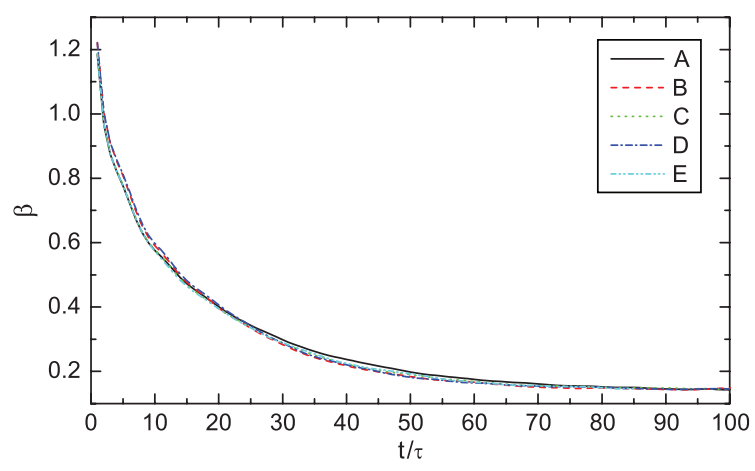

Fig. 18. Same as Figure 17, except that the randomness in the coupling constants of $H_{\mathrm{SE}}$ or $H_{\mathrm{E}}$, or the randomness in the initial state of the environment, are different in the curves $A-E$. It is clear that the time evolution of the effective temperature $b(t)$ is not sensitive to the different random values of the coupling constants or the initial state of the environment if they follow the same type of random distribution. 


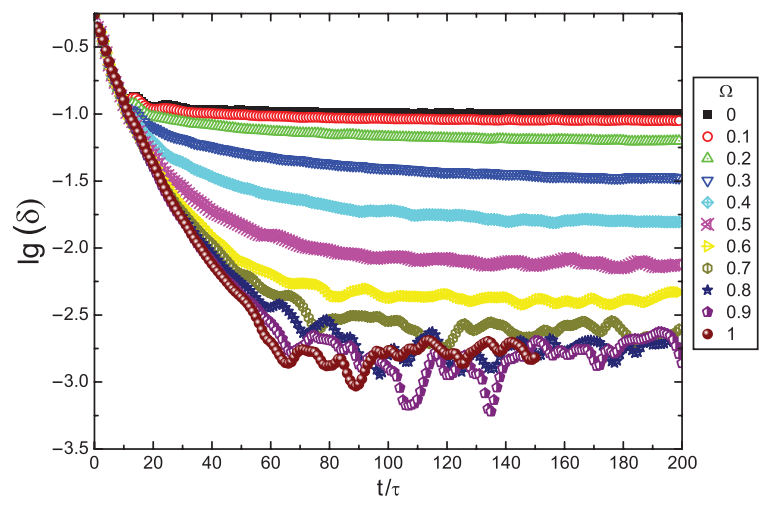

Fig. 19. Same as Figure 17 except that the range of the coupling strength $(\Omega)$ is different in the environment of each curve.

The time evolution of $\delta(t)$ for the same systems but with different topological structures (connectivity $K$ ) in the environment are shown in Figure 20. Increasing the connectivity in the environment will increase the energy resolution and make the dynamics in the environment more complicated, which enhances the approach to the canonical distribution of the system. This is quite different from the effect of the topological structure on the decoherence shown in Figure 16, where as soon as there is frustrated interaction within the system $(K>0)$, the decoherence of the quantum system is quite similar for different topological structures.

More results for the system with different symmetries and connectivities but with the same type of environments $\left(H_{\mathrm{E}}\right)$ and the same type of interactions $\left(H_{\mathrm{SE}}\right)$ are shown in Figure 22. The systems used are a XY-ring, a Heisenberg-ring, an Ising-ring, a Heisenberg-triangularlattice, and a spin glass. From the results represented in Figure 22, it is clear that independent of the internal symmetries and the connectivity of the system, and independent of the initial state of the whole system, all systems

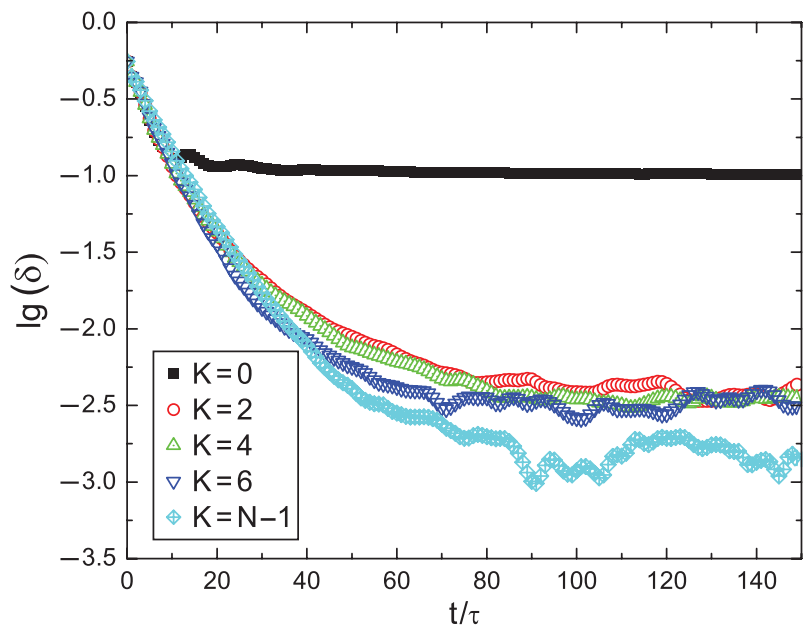

Fig. 20. Same as Figure 17 except that the topological structure (connectivity $K$ ) of the environment is different. relax to a state with full decoherence, except case f. The main difference between case $\mathbf{f}$ and the other cases is the initial state of the environment. Since the environment in our model is a highly frustrated system, the LDOS of the whole system covers the whole spectrum of the energy space, no matter the initial state of the environmental spins is all spins up or all spins down, or random spins up and down, or a random superposition of all the states in the spin up-down basis. But if the environment is prepared in the ground state or near ground state, then the LDOS of the whole system becomes more sharply peaked, see $\mathbf{b}$ and $\mathbf{f}$ in Figure 23. Up to a trivial normalization factor, the LDOS curve for case $\mathbf{b}$ is indistinguishable from the density of states (data not shown) calculated from the solution of the TDSE using the technique described in Ref. [93]. This suggests that if the environment starts from the random superposition of all its energy eigenstates, all states of the whole system may participate in the decoherence/relaxation process. In contrast, the LDOS curve for case $\mathbf{f}$ has a very small overlap with the density of states. Therefore, starting with an environment in the ground state, only a relatively small number of states participates in the decoherence process, as confirmed by the results for $\sigma(t)$ shown in Figure 22(f).

One should also notice that in case $\mathbf{b}, \sigma$ vanishes exponentially with time, whereas in the other cases $(\mathbf{a}, \mathbf{c}, \mathbf{d}, \mathbf{e})$, $\sigma$ initially increases and then vanishes exponentially with time, due to the entanglement between the system and the environment. This observation is in concert with our earlier work. ${ }^{23-25}$ Furthermore, in all cases except $\mathbf{f}$, the system always relaxes to a canonical distribution $(\delta \rightarrow 0)$ as soon as it is in the state with full decoherence $(\sigma \rightarrow 0)$, indicating that the time of decoherence $\left(T_{2}\right)$ and the time of thermalization $\left(T_{1}\right)$ is almost the same. In agreement with the results depicted in Fig. 17, the decoherence time $T_{2}$ is shorter than the typical time scale $T_{1}$ on which the system and the environment exchange energy. Note that in contrast to the cases considered in the theory of nuclear magnetic resonance, in most of our simulations, $H_{\mathrm{S}}, H_{\mathrm{E}}$ and $H_{\mathrm{SE}}$ are

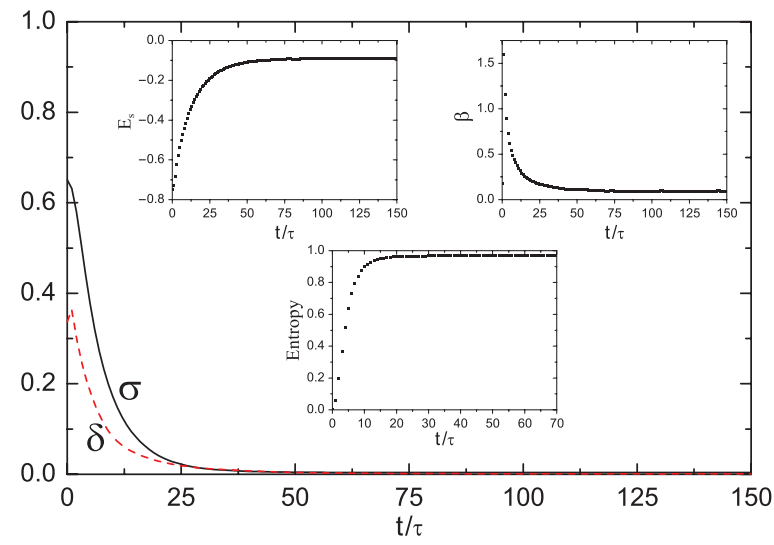

Fig. 21. Same as Figure 17 except that $n_{\mathrm{S}}=5$ and $n=17$. 
(a)

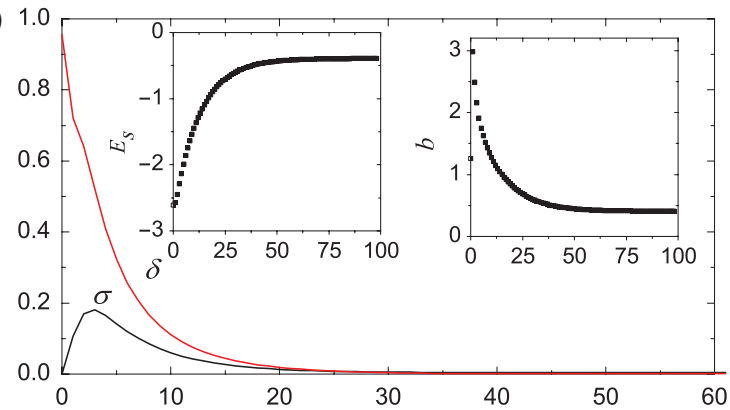

(d)

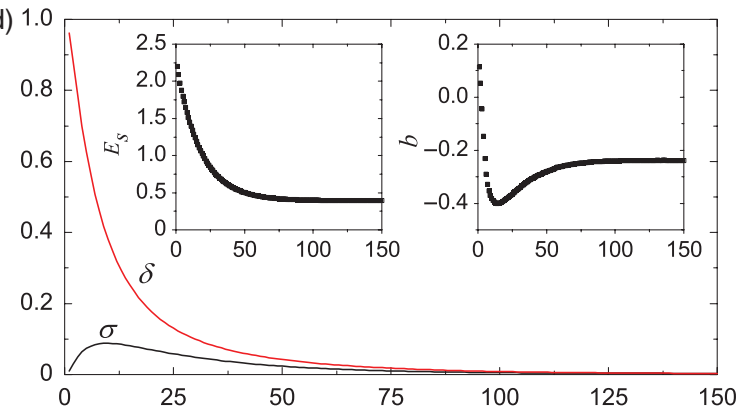

(b) 1.0

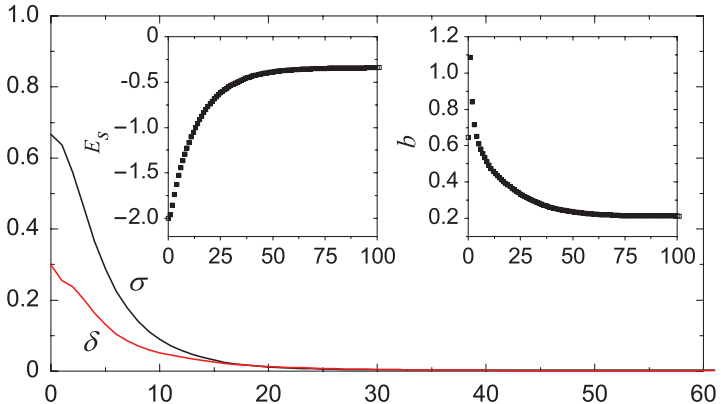

(e) 1.0

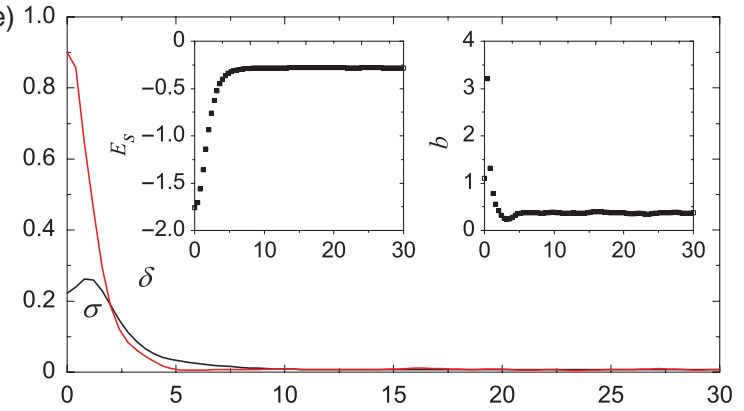

(c)

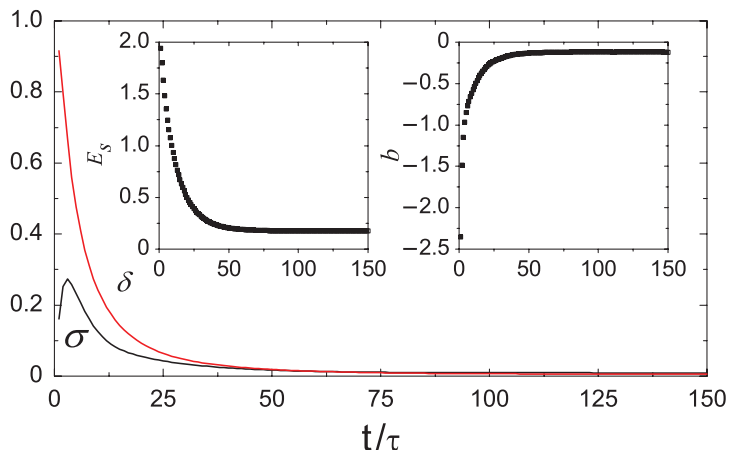

(f) 1.0

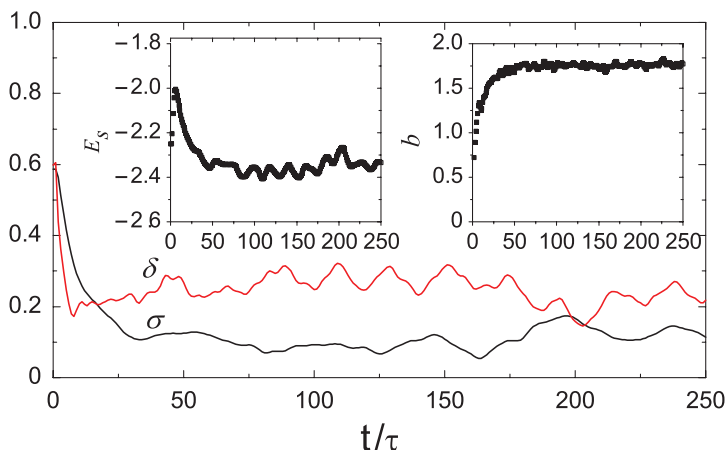

Fig. 22. Simulation results for the energy $E_{\mathrm{S}} \equiv E_{\mathrm{S}}(t)$, the effective inverse temperature $b \equiv b(t)$, its variance $\delta \equiv \delta(t)$, and the deviation from a diagonal matrix $\sigma \equiv \sigma(t)$ as obtained by the solution of the TDSE for a variety of different systems $S$ coupled to a spin glass environment $H_{\mathrm{E}}$ via a Heisenberg-type interaction $H_{\mathrm{SE}}$. The systems used are (a): XY-ring, (b) and (f) Heisenberg-ring, (c) Ising-ring, (d) Heisenberg-triangular-lattice, and (e) spin glass. The initial states of the whole system are (a) $\mid$ GROUND $\rangle_{\mathrm{S}} \otimes|R A N D O M\rangle_{\mathrm{E}}$, (b) $|U D\rangle_{\mathrm{S}} \otimes|R A N D O M\rangle_{\mathrm{E}}$, (c) $|U U\rangle_{\mathrm{S}} \otimes|R R\rangle_{\mathrm{E}}$, (d) $|U U\rangle_{\mathrm{S}} \otimes|R A N D O M\rangle_{\mathrm{E}}$, (e) $\mid$ GROUND $\rangle_{\mathrm{S}} \otimes|U D\rangle_{\mathrm{E}}$, and (f) $|\widetilde{U D}\rangle_{\mathrm{S}} \otimes|G R O U N D\rangle_{\mathrm{E}}$. The numbers of spins in the system are $n_{\mathrm{S}}=8$ for cases (a)-(c) and $n_{\mathrm{S}}=6$ for cases (d)-(f). The number of spins in the environment is $n=16$ for all cases. The model parameters are $J=-1, \Delta=0.3$ and $\Omega=1$, except for case (e) in which $\Delta=1$.

comparable so the standard perturbation derivation of $\sigma$ and $E$ does not work.

The negative temperature $(b<0)$ is also observed in $\mathbf{c}$ and $\mathbf{d}$. In fact, as the temperature $T$ is defined as

$$
\frac{1}{T}=\frac{d S}{d E}
$$

where $S$ and $E$ are the entropy and energy. In a quantum spin system, the entropy may decrease when the energy increases. For example, the states with all spins up and all spins down have the same entropy, but can have totally different energy. Suppose there is no interaction between $N$ ferromagnetic spins $(J>0)$, and there is a uniform magnetic filed applied on the $Z+$ direction, then the state with all spins up is the ground state, and with all spins down is the eigenstate with highest energy. Changing the magnetization of the states from $M_{\max }=N / 2$ to $M_{\min }=-N / 2$ will change the sign of the temperature $T(M)$ at the point $M=0$, that is, $T>0$ when $M>0$ and $T<0$ when $M<0$.

\subsection{Summary}

We have shown that if we have a system that interacts with an environment and the whole system forms a closed quantum system that evolves in time according to the TDSE, then a frustrated environment with a random distribution in the energy basis will lead to the full decoherence of the system. Furthermore, if the system and environment can exchange energy, the range of energies of the environment is large compared to the range of energies of the 


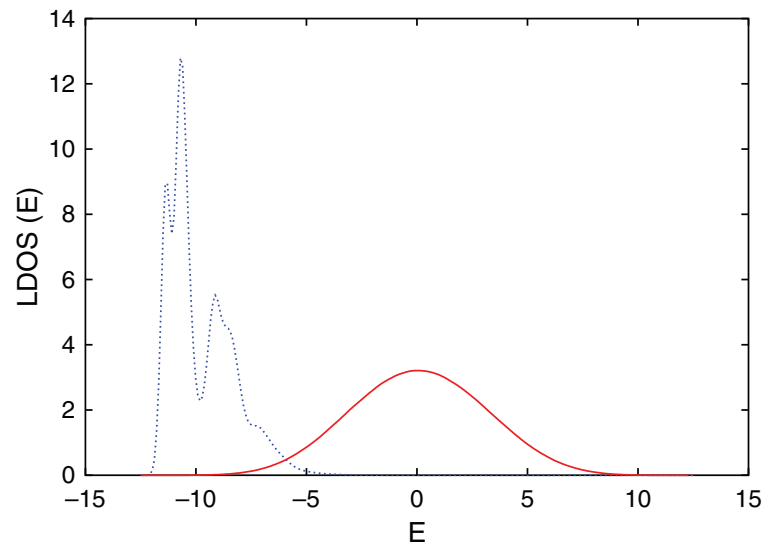

Fig. 23. Simulation results for the local density of states as a function of the energy. Solid line: Case corresponding to Figure 22(b). The initial state is $|U D\rangle_{\mathrm{S}} \otimes|R A N D O M\rangle_{\mathrm{E}}$; Dashed line: Case corresponding to Figure 22(f). The initial state is $|U D\rangle_{\mathrm{S}} \otimes|G R O U N D\rangle_{\mathrm{E}}$. Reprinted with permission from [26], S. Yuan et al., J. Phys. Soc. Jpn. 78, 094003 (2009). () 2009.

system, then the mixed state of the system is a canonical distribution.

\section{CONCLUSION AND DISCUSSION}

The results presented here have been obtained from an $a b$ initio numerical solution of the TDSE in the absence of, for instance, dissipative mechanisms, and demonstrate that the existence of the microcanonical distribution (in each eigenenergy subspace) and the canonical ensemble is a direct consequence of quantum dynamics.

We emphasize that our conclusion does not rely on time averaging of observables, in concert with the fact that real measurements of thermodynamic properties yield instantaneous, not time-averaged, values. Furthermore and perhaps a little counter intuitive, our results show that relatively small environments $(\approx 20$ spins) are sufficient to drive the system (2-8 spins) to thermal equilibrium and that there is no need to assume that the interaction between the system and environment is weak, as is usually done in kinetic theory. Note that even if most cases shown in this review clearly indicate a full decoherence of the quantum system, it does not mean that the condition of full decoherence is a nontrivial requirement.

To conclude, we find that:

(1) Frustration of the interactions, either within the environment or between the system and the environment, enhances the decoherence of the quantum system.

(2) The quantum system more easily evolves into its ground state when the latter is more degenerate or less entangled, or has certain additional integrals of motion.

(3) The distribution of the state of a quantum system is the microcanonical or canonical ensemble only if the system is in a fully decoherent state.
(4) The restriction of a fully decoherent state to be a microcanonical ensemble per eigenenergy subspace is the presence of an additional integral of motion except a conserved energy. For example, a conserved magnetization of the quantum system prevents parts of the degenerate eigenstates to be the pointer states.

(5) The restriction of a microcanonical ensemble to be a canonical ensemble is the presence of an additional integral of motion, so that the energy of each subspace is conserved.

(6) The distributions in quantum statistical mechanics, such as the microcanonical and canonical distributions, are the direct consequence of quantum dynamics.

Finally we want to discuss the second law of thermodynamics in quantum systems.

The second law of thermodynamics states that the entropy of an isolated system which is not in equilibrium tends to increase over time, approaching a maximum value at equilibrium. In quantum mechanics, the state of a closed quantum system is always a pure state and therefore its entropy is a constant (zero). It is thus clear that the second law of thermodynamics is not valid in a closed quantum system. If a quantum system starts to interact with an environment, its entropy increases from zero but may not reach a maximum value at equilibrium. The dynamics of the whole system could be periodical and therefore the time evolution of the states could be reversible.

Our numerical results show that if the quantum system becomes a classical mixed state, then the time evolution becomes irreversible and the entropy becomes stable when it reaches the maximum value. In fact, the state with the microcanonical distribution in each eigenenergy subspace or in the canonical ensemble has the maximum entropy within all possible states that the system could be. For a quantum system with a certain energy and a fixed number of particles it is such that, if there is a conservation law to restrict some eigenstates in the reduced density matrix, then the state with maximum entropy corresponds to the one with all the accessible degenerate states having the same weigth distribution, i.e., a microcanonical distribution per eigenenergy subspace. On the other hand, if there is no such restriction on the eigenstates, then the canonical ensemble is the state with maximum entropy, as proved by Jaynes in Ref. [97].

We may conclude that the validity of the second law of thermodynamics in quantum mechanics is related to the decoherence process of the quantum system. If a quantum system becomes classical under the influence of the environment, then its entropy will increase until it reaches a maximum value of all possible mixed states, i.e., the microcanonical distribution per eigenenergy subspace or the canonical ensemble. If a quantum system cannot evolve into a stable mixed state, then its entropy will not always follow the second law of thermodynamics. 
Acknowledgment: It is a pleasure to thank $\mathrm{H}$. De Raedt, M. I. Katsnelson, S. Miyashita, K. Michielsen, F. Jin and S. Zhao for many helpful discussions. The support by the Stichting Fundamenteel Onderzoek der Materie (FOM) and the Netherlands National Computing Facilities foundation (NCF) are acknowledged.

\section{References}

1. R. V. Jensen and R. Shankar, Phys. Rev. Lett. 54, 1879 (1985).

2. H. D. Zeh, Foundation of Physics 1, 69 (1970).

3. D. Giulini, E. Joos, C. Kiefer, J. Kupsch, I.-O. Stamatescu, and H. D. Zeh, Decoherence and the Appearance of a Classical World in Quantum Theory, Springer, Berlin (1996).

4. W. H. Zurek, Phys. Rev. D 24, 1516 (1981).

5. W. H. Zurek, Phys. Rev. D 26, 1862 (1982).

6. W. H. Zurek, Physics Today 44, 36 (1991).

7. W. H. Zurek, Phil. Trans. R. Soc. Lond. A 356, 1793 (1998).

8. W. H. Zurek, Decoherence and the Transition from Quantum to Classical-Revisited, Los Alamos Science Number 27 (2002).

9. W. H. Zurek, Rev. Mod. Phys. 75, 715 (2003).

10. W. H. Zurek, Nat. Phys. 5, 181 (2009).

11. J.-P. Paz and W. H. Zurek, Phys. Rev. Lett. 82, 5181 (1999).

12. M. Nielsen and I. Chuang, Quantum Computation and Quantum Information, Cambridge University Press, Cambridge (2000).

13. B. E. Kane, Nature 393, 133 (1998).

14. M. N. Leuenberger and D. Loss, Nature 410, 789 (2001).

15. B. Trauzettel, D. V. Bulaev, D. Loss, and G. Burkard, Nat. Phys. 3, 192 (2007).

16. J. von Neumann, Mathematical Foundations of Quantum Mechanics, Princeton University Press, Princeton (1955).

17. R. P. Feynman and F. L. Vernon, Ann. Phys. (N.Y.) 24, 118 (1963).

18. A. J. Leggett, S. Chakravarty, A. T. Dorsey, M. P. A. Fisher, A. Garg, and W. Zwerger, Rev. Mod. Phys. 59, 1 (1987).

19. N. V. Prokof'ev and P. C. E. Stamp, Rep. Prog. Phys. 63, 669 (2000).

20. V. V. Dobrovitski, H. A. De Raedt, M. I. Katsnelson, and B. N. Harmon, Phys. Rev. Lett. 90, 210401 (2003).

21. J. Lages, V. V. Dobrovitski, M. I. Katsnelson, and B. N. Harmon, Phys. Rev. E 72, 026225 (2005).

22. A. Melikidze, V. V. Dobrovitski, H. A. De Raedt, M. I. Katsnelson, and B. N. Harmon, Phys. Rev. B 70, 014435 (2004).

23. S. Yuan, M. I. Katsnelson, and H. De Raedt, JETP Lett. 84, 99 (2006).

24. S. Yuan, M. I. Katsnelson, and H. De Raedt, Phys. Rev. A 75, 052109 (2007).

25. S. Yuan, M. I. Katsnelson, and H. De Raedt, Phys. Rev. B 77, 184301 (2008).

26. S. Yuan, M. I. Katsnelson, and H. De Raedt, J. Phys. Soc. Jpn. 78, 094003 (2009).

27. J. van Wezel, J. van den Brink, and J. Zaanen, Phys. Rev. Lett. 94, 230401 (2005).

28. X. Z. Yuan, H.-S. Goan, and K. D. Zhu, Phys. Rev. B 75, 045331 (2007).

29. X. Z. Yuan and K. D. Zhu, Europhys. Lett. 69, 868 (2005).

30. Y. Gao and S. J. Xiong, Phys. Rev. A 71, 034102 (2005).

31. W. Zhang, V. V. Dobrovitski, K. A. Al-Hassanieh, E. Dagotto, and B. N. Harmon, Phys. Rev. B 74, 205313 (2006).

32. W. Zhang, N. Konstantinidis, K. A. Al-Hassanieh, and V. V. Dobrovitski, J. Phys. Cond. Matter 19, 083202 (2007).

33. C. M. Dawson, A. P. Hines, R. H. McKenzie, and G. J. Milburn, Phys. Rev. A 71, 052321 (2005).

34. D. Rossini, T. Calarco, V. Giovannetti, S. Montangero, and R. Fazio, Phys. Rev. A 75, 032333 (2007).
35. L. Tessieri and J. Wilkie, J. Phys. A, Math. Gen. 36, 12305 (2003).

36. S. Camalet and R. Chitra, Phys. Rev. B 75, 094434 (2007).

37. D. D. B. Rao, V. Ravishankar, and V. Subrahmanyam, Phys. Rev. A 75, 052338 (2007).

38. A. Relano, J. Dukelsky, and R. A. Molina, Phys. Rev. E 76, 046223 (2007).

39. V. V. Dobrovitski, M. I. Katsnelson, and B. N. Harmon, Phys. Rev. Lett. 84, 3458 (2000).

40. S. O. Skrøvseth, Europhys. Lett. 76, 1179 (2006).

41. G.-F. Zhang, J.-Q. Liang, G.-E. Zhang, and Q.-W. Yan, Eur. Phys. J. D 32, 409 (2005).

42. Y. Hamdouni, M. Fannes, and F. Petruccione, Phys. Rev. B 73, 245323 (2006).

43. K. Saito, S. Takasue, and S. Miyashita, Phys. Rev. Lett. 65, 1243 (1996).

44. P. Bocchieri and A. Loinger, Phys. Rev. 114, 948 (1959).

45. R. V. Jensen and R. Shankar, Phys. Rev. Lett. 54, 1879 (1985).

46. H. Tasaki, Phys. Rev. Lett. 80, 1373 (1998).

47. S. Popescu, A. J. Short, and A. Winter, Nat. Phys. 2, 754 (2006)

48. M. Rigol, V. Dunjko, and M. Olshanii, Nature 452, 854 (2008).

49. S. Goldstein, J. L. Lebowitz, R. Tumulka, and N. Zangh, Phys. Rev. Lett. 96, 050403 (2006).

50. P. Reimann, Phys. Rev. Lett. 99, 160404 (2007).

51. P. Reimann, J. Stat. Phys. 132, 921 (2008).

52. J. Gemmer and M. Michel, Europhys. Lett. 73,1 (2006).

53. J. Gemmer and M. Michel, Eur. Phys. J. B 53, 517 (2006).

54. M. A. Cazalilla, Phys. Rev. Lett. 97, 156403 (2006).

55. M. Rigol, A. Muramatsu, and M. Olshanii, Phys. Rev. A 74, 053616 (2006).

56. M. Rigol, V. Dunjko, V. Yurovsky, and M. Olshanii, Phys. Rev. Lett. 98, 050405 (2007).

57. M. Eckstein and M. Kollar, Phys. Rev. Lett. 100, 120404 (2008).

58. M. Cramer, A. Flesch, I. P. McCulloch, U. Schollwock, and J. Eisert, Phys. Rev. Lett. 101, 063001 (2008).

59. M. Cramer, C. M. Dawson, J. Eisert, and T. J. Osborne, Phys. Rev. Lett. 100, 030602 (2008).

60. A. Flesch, M. Cramer, I. P. McCulloch, U. Schollwock, and J. Eisert, Phys. Rev. A 78, 033608 (2008).

61. M. Esposito and P. Gaspard, Phys. Rev. E 68, 066113 (2003).

62. M. Merkli and I. M. Sigal, Phys. Rev. Lett. 98, 130401 (2007).

63. G. Benenti, G. Casati, and D. L. Shepelyansky, Eur. Phys. J. D 17, 265 (2001).

64. R. Balescu, Equilibrium and Nonequilibrium Statistical Mechanics, Wiley, New York (1975).

65. J. von Neumann, Mathematical Foundations of Quantum Mechanics, Princeton University Press, Princeton (1955).

66. L. E. Ballentine, Quantum Mechanics, A Modern Development, World Scientific, Singapore (2003).

67. M. Znidaric and T. Prosen, J. Phys. A: Math. Gen. 36, 2463 (2003).

68. T. Prosen, T. H. Seligman, and M. Znidaric, Prog. Theor. Phys. Suppl. 150, 200 (2003).

69. F. M. Cucchietti, D. A. R. Dalvit, J. P. Paz, and W. H. Zurek, Phys. Rev. Lett. 91, 210403 (2003).

70. H. Fujisaki, T. Miyadera, and A. Tanaka, Phys. Rev. E 67, 066201 (2003)

71. T. Gorin, T. Prosen, T. H. Seligman, and M. Znidaric, Physics Reports, 435, 33 (2006)

72. L. Amico, R. Fazio, A. Osterloh, and V. Vedral, Rev. Mod. Phys. 80,517 (2008)

73. K. Binder and A. P. Young, Rev. Mod. Phys. 58, 801 (1986).

74. M. Mezard, G. Parisi, and M. A. Virasoro, Spin Glass Theory and Beyond, World Scientific, Singapore (1987).

75. H. Tal-Ezer and R. Kosloff, J. Chem. Phys. 81, 3967 (1984).

76. C. Leforestier, R. H. Bisseling, C. Cerjan, M. D. Feit, R. Friesner, A. Guldberg, A. Hammerich, G. Jolicard, W. Karrlein, H.-D. 
Meyer, N. Lipkin, O. Roncero, and R. Kosloff, J. Comp. Phys. 94, 59 (1991).

77. T. Iitaka, S. Nomura, H. Hirayama, X. Zhao, Y. Aoyagi, and T. Sugano, Phys. Rev. E 56, 1222 (1997).

78. V. V. Dobrovitski and H. De Raedt, Phys. Rev. E 67, 056702 (2003).

79. H. F. Trotter, Proc. Am. Math. Soc. 10, 545 (1959).

80. M. Suzuki, S. Miyashita, and A. Kuroda, Prog. Theor. Phys. 58, 1377 (1977).

81. M. Suzuki, J. Math. Phys. 26, 601 (1985).

82. M. Suzuki, J. Math. Phys. 32, 400 (1991).

83. H. De Raedt and B. De Raedt, Phys. Rev. A 28, 3575 (1983).

84. H. De Raedt, Comp. Phys. Rep. 7, 1 (1987).

85. H. De Raedt and P. de Vries, Z. Phys. B 77, 243 (1989).

86. P. de Vries and H. De Raedt, Phys. Rev. B 47, 7929 (1993).

87. K. Michielsen, H. De Raedt, J. Przeslawski, and N. García, Phys. Rep. 304, 89 (1998)

88. H. De Raedt, A. H. Hams, K. Michielsen, and K. De Raedt, Comp. Phys. Comm. 132, 1 (2000).
89. H. De Raedt and K. Michielsen, Computational methods for simulating quantum computers, Handbook of Theoretical and Computational Nanotechnology, Quantum and Molecular Computing, Quantum Simulations, edited by M. Rieth and W. Schommers, American Scientific Publisher, Los Angeles (2006), Vol. 3, Chap. 1, pp. 2-48.

90. M. Krech, A. Bunker, and D. P. Landau, Comp. Phys. Comm. 111, 1 (1998).

91. S. Yuan, H. De Raedt, and S. Miyashita, J. Phys. Soc. Jpn. 75, 084703 (2006)

92. S. Yuan, H. De Raedt, and S. Miyashita, Phys. Rev. B 75, 184305 (2007)

93. A. H. Hams and H. De Raedt, Phys. Rev. E 62, 4365 (2000).

94. W. K. Wootters, Phys. Rev. Lett. 80, 2245 (1998).

95. S. Hill and W. K. Wootters, Phys. Rev. Lett. 78, 5022 (1997)

96. A. Abragam, The Principles of Nuclear Magnetism, Clarendon Press, Oxford (1961).

97. E. T. Jaynes, Phys. Rev. 106, 620 (1957).

Received: 21 March 2010. Accepted: 26 May 2010. 